\title{
Semaglutide attenuates seizure severity and ameliorates cognitive dysfunction by blocking the NLR family pyrin domain containing 3 inflammasome in pentylenetetrazole-kindled mice
}

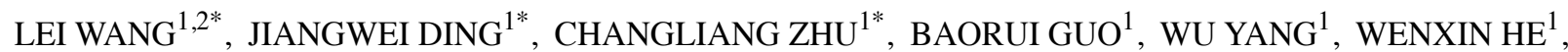 \\ XINXIAO LI ${ }^{1,3}$, YANGYANG WANG ${ }^{1,2}$, WENCHAO LI ${ }^{1,2}$, FENG WANG $^{4}$ and TAO SUN ${ }^{1}$ \\ ${ }^{1}$ Ningxia Key Laboratory of Cerebrocranial Disease, Incubation Base of National Key Laboratory, Ningxia Medical University, \\ Yinchuan, Ningxia $750000 ;{ }^{2}$ Department of Neurosurgery, The First Affiliated Hospital of Xinxiang Medical University, \\ Weihui, Henan 453100; ${ }^{3}$ Department of Neurosurgery, The Fifth Affiliated Hospital of Zhengzhou University, \\ Zhengzhou, Henan 450000; ${ }^{4}$ Department of Neurosurgery, The First Affiliated Hospital, \\ Zhejiang University School of Medicine, Hangzhou, Zhejiang 310000, P.R. China
}

Received August 5, 2021; Accepted October 5, 2021

DOI: $10.3892 /$ ijmm.2021.5052

\begin{abstract}
Epilepsy comorbidities and anti-epileptic drugs (AEDs) are currently the main limitations of epilepsy treatment. Semaglutide is a glucagon like peptide-1 analogue that has entered the market as a new once-weekly drug for type II diabetes. The aim of the present study was to investigate the functions of semaglutide in epilepsy and inflammation models, in order to investigate its potential mechanism. In vitro, an inflammation model was established using lipopolysaccharide (LPS) and nigericin stimulation in BV2 cells. In vivo, chronic epilepsy model mice were generated using a pentylenetetrazole (PTZ) kindling method. BV2 cell proliferation was assessed using the Cell Counting Kit-8. The effects of semaglutide on NLR family pyrin domain containing 3 (NLRP3) inflammasome activation and inflammatory cytokine secretion were determined using western blotting (WB) and ELISA. A lactate dehydrogenase (LDH) assay kit was used to detect the effect of semaglutide on LDH release. Electrocorticography and the modified Racine scale were used to assess seizure severity.
\end{abstract}

Correspondence to: Professor Tao Sun, Ningxia Key Laboratory of Cerebrocranial Disease, Incubation Base of National Key Laboratory, Ningxia Medical University, 1160 Shengli Street, Yinchuan, Ningxia 750000, P.R. China

E-mail: suntao_nxmu@163.com

Professor Feng Wang, Department of Neurosurgery, The First Affiliated Hospital, Zhejiang University School of Medicine, 79 Qingchun Road, Hangzhou, Zhejiang 310000, P.R. China

E-mail: nxwwang@163.com

${ }^{*}$ Contributed equally

Key words: epilepsy, glucagon like peptide-1, semaglutide, NLR family pyrin domain containing 3 inflammasome, neuroprotection, cognitive dysfunction
Cognitive function was evaluated with behavioral assessment. Morphological changes in the hippocampus were observed with Nissl staining. Double immunofluorescence staining for NeuN and Iba-1, WB and immunofluorescence analysis of apoptosis-related proteins were used to evaluate neuronal apoptosis. The NLRP3 inflammasome was assessed by reverse transcription-quantitative PCR, WB and immunofluorescence staining, and inflammatory cytokine release was evaluated by WB analysis in the hippocampus of C57/BL6J model mouse. Semaglutide attenuated the LPS- and nigericin-induced inflammatory response and LDH release by blocking NLRP3 inflammasome activation in BV2 cells. Moreover, semaglutide decreased seizure severity, alleviated hippocampal neuronal apoptosis, ameliorated cognitive dysfunction, blocked NLRP3 inflammasome activation and decreased inflammatory cytokine secretion in PTZ-kindled mice. These results indicated that semaglutide reduced seizure severity, exerted neuroprotective effects and ameliorated cognitive dysfunction, possibly via inhibition of NLRP3 inflammasome activation and inflammatory cytokine secretion. Semaglutide may therefore be a novel, promising adjuvant therapeutic for epilepsy and its associated comorbidities.

\section{Introduction}

Epilepsy is a common and widespread disease of the central nervous system (CNS) (1); $\sim 5$ million individuals are diagnosed annually $(2,3),>65$ million are affected by epilepsy and epileptic comorbidities worldwide (4), and $\sim 15-30 \%$ of patients with epilepsy experience epilepsy-related comorbidities (5). Neuronal injury and neuroinflammation caused by long-term recurrent seizures are characteristics of epilepsy that further result in behavioral disorders (6,7). Anti-epileptic drug (AED) therapy remains the preferred treatment for epilepsy, and although 30 types of AEDs with diverse molecular targets are available, numerous challenges are associated with their use (8), among which AED resistance and side effects are the primary issues $(9,10)$. In addition, $\sim 30 \%$ of patients with epilepsy fail 
to respond to AEDs, and long-term use affects cognitive function and causes psychological impairments $(8,11)$. Therefore, research into novel AEDs with greater effectiveness and fewer side effects is urgently required.

Previous studies have suggested that neuroinflammatory processes play a key role in epileptogenesis. Seizures cause neuroinflammation, which in turn aggravates epilepsy $(12,13)$. The NLRP3 inflammasome results from the assembly of NLR family pyrin domain containing 3 (NLRP3), apoptosis-associated speck-like protein (ASC) and caspase-1 (14), and promotes the secretion of inflammatory cytokines, influencing the pathophysiology of epilepsy (14). Additionally, in animal and clinical epilepsy-related research, increased levels of the NLRP3 inflammasome and neuroinflammatory cytokines have been detected in hippocampal tissues $(15,16)$, suggesting their close association with epileptogenesis. Moreover, numerous studies have reported that inhibiting NLRP3 activation and inflammatory cytokine secretion decreases to occurrence of epileptic seizures and ameliorates cognitive dysfunction $(17,18)$. These findings suggest that inhibition of NLRP3 inflammasome activity and the associated cascade reactions may be a promising approach for adjuvant treatment of epilepsy.

Initially, glucagon like peptide-1 (GLP-1) was widely regarded as a peripheral incretin hormone. Natural GLP-1 has a short half-life and is quickly degraded by proteases, which promotes GLP-1 analogue generation (19). Originally, GLP-1 analogues were used as second-line hypoglycemic drugs, and further research has demonstrated that GLP-1 exerts anti-inflammatory and neuroprotective effects, as well as the ability to ameliorate cognitive dysfunction (20). Numerous studies have also demonstrated that GLP-1 analogues reduce tissue apoptosis by suppressing NLRP3 inflammasome activation and downstream inflammatory cytokine secretion $(21,22)$. In the brain, preproglucagon neurons synthesize GLP-1; subsequently, GLP-1 is distributed in the nucleus of the solitary tract, where it functions as a neuropeptide. GLP-1 receptors are widely expressed in various structures, such as the brainstem, cerebral cortex and hippocampus $(23,24)$. Moreover, an increasing number of studies have suggested that GLP-1 signaling may serve as a potential drug target for the management of various neurological disorders, including epilepsy and its associated comorbidities (20,25). Semaglutide entered the market in 2017 as a novel once-weekly GLP-1 analogue for the treatment of type II diabetes (26). In addition, semaglutide has been shown to reduce apoptosis and ameliorate cognitive impairment in cerebrovascular disease (27), Alzheimer's disease (AD) (28) and Parkinson's disease (PD) (29). Moreover, a number of reports have indicated that patients with $\mathrm{AD}$ and AD model mice are markedly susceptible to unprovoked seizures (30), and AD-related pathological changes have been detected in epileptic foci (31). However, limited research has been conducted on the potential role of semaglutide in epileptogenesis.

Therefore, the aim of the present study was to investigate whether semaglutide affects seizure severity and epilepsy-related cognitive impairment. The results obtained herein, including those of mouse and cellular models, may support further investigation into semaglutide use in patients with epilepsy and associated comorbidities.

\section{Materials and methods}

Cell lines and culture. BV2 cells (Guangzhou Cellcook Biotech Co., Ltd.) were maintained in DMEM supplemented with $10 \%$ fetal bovine serum (FBS) (both Gibco; Thermo Fisher Scientific, Inc.) and 1\%; penicillin/streptomycin (Beijing Solarbio Science \& Technology Co., Ltd.) and cultured at $37^{\circ} \mathrm{C}$ with $5 \% \mathrm{CO}_{2}$.

The in vitro study protocols are outlined in Fig. 1A. BV2 cells were assigned to four groups as follows: i) The control group, treated with $1 \%$ DMSO; ii) the semaglutide group, treated with $900 \mathrm{nM}$ semaglutide (purity, $\geq 98 \%$; cat. no. HY-114118; MedChemExpress); iii) the lipopolysaccharide (LPS; cat. no. L2630; Sigma-Aldrich; Merck KGaA) + nigericin (cat. no. HY-100381; MedChemExpress) group, treated with $1 \mu \mathrm{g} / \mathrm{ml}$ LPS (32) and $10 \mu \mathrm{M}$ nigericin (33); and iv) the semaglutide + LPS + nigericin group, treated with $900 \mathrm{nM}$ semaglutide followed by $1 \mu \mathrm{g} / \mathrm{ml}$ LPS and $10 \mu \mathrm{M}$ nigericin.

Cellular proliferation. Cellular proliferation was evaluated using the Cell Counting Kit-8 (CCK-8) assay (Dojindo Laboratories, Inc.). BV2 cells were seeded into 96-well plates ( $1 \times 10^{4}$ cells/well) for $12 \mathrm{~h}$, and then treated with different doses $(300,600,900$ or $1,000 \mathrm{nM})$ of semaglutide for $20 \mathrm{~h}$ at $37^{\circ} \mathrm{C}$. A blank well with culture medium was used to detect background, and five replicate wells were established per group. After $20 \mathrm{~h}, 10 \% \mathrm{CCK}-8$ reagent $(100 \mu \mathrm{l})$ was added to each well, and the cells were cultured at $37^{\circ} \mathrm{C}$ in $5 \% \mathrm{CO}_{2}$ for $1 \mathrm{~h}$. The absorbance value was recorded at $450 \mathrm{~nm}$ using a microplate reader (BioTek Instruments, Inc.), and the optical density (OD) value was recorded for evaluation.

ELISA. The supernatants of each group of BV2 cells were harvested, and the levels of IL-1 $\beta$ (cat. no. JL18442), IL-18 (cat. no. JL20253), IL-6 (cat. no. JL20268) and TNF- $\alpha$ (cat. no. JL10484) were evaluated using the associated ELISA kits (Jianglai Industrial Limited By Share, Ltd.) according to the manufacturer's instructions. A microplate reader was used to record the absorbance and OD values at $450 \mathrm{~nm}$.

Lactate dehydrogenase $(\mathrm{LDH})$ assay. Each group of BV2 cell supernatants was collected, and the LDH concentration was determined using an LDH assay kit (Nanjing KeyGen Biotech Co., Ltd.) according to the manufacturer's instructions. The absorbance at $450 \mathrm{~nm}$ was determined using a microplate reader, and the OD value was recorded for further evaluation.

Western blotting (WB). Total protein was extracted from BV2 cells and hippocampal tissues using a Whole Protein Extraction kit, and the protein concentrations were determined using a BCA Protein Assay kit (both Nanjing KeyGen Biotech Co., Ltd.). Equal amounts of protein (50-60 $\mu \mathrm{g}$ per lane) were separated by $10 \%$ SDS-PAGE and then transferred to $0.22-\mathrm{mm}$ PVDF membranes (MilliporeSigma). To block non-specific binding, $4 \%$ bovine serum albumin (BSA) was applied at room temperature (RT) for $1.5 \mathrm{~h}$, after which the membrane was incubated with the following primary antibodies for $17 \mathrm{~h}$ at $4^{\circ} \mathrm{C}$ : Anti-NLRP3 (cat. no. bs-10021R), anti-ASC (cat. no. bs-6741R), anti-IL-1 $\beta$, (cat. no. bs-0812R) and anti-caspase-1p20 (cat. 

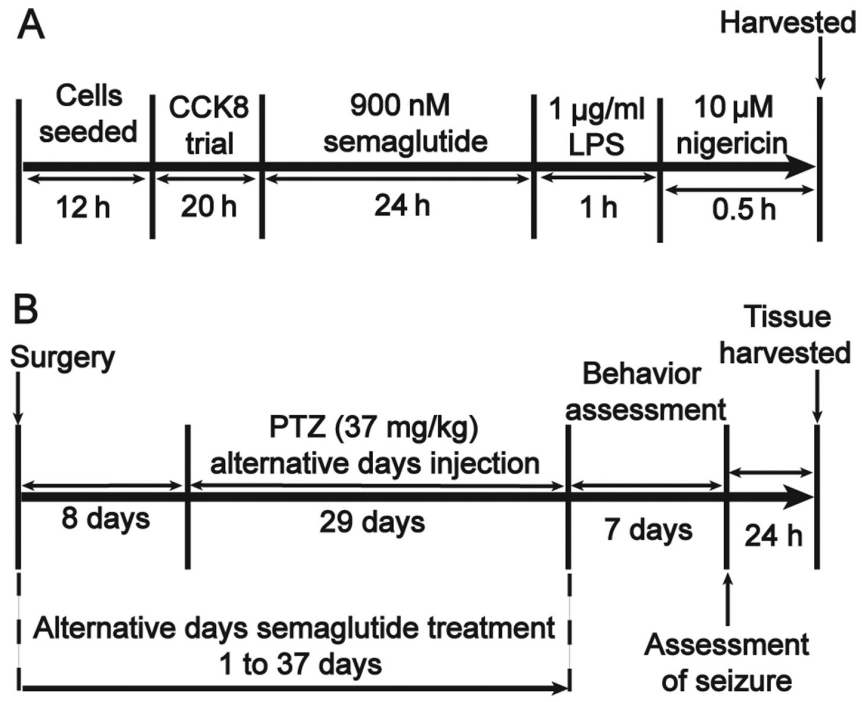

Figure 1. Study experimental protocols. (A) BV2 cell treatment flowchart. (B) Mouse treatment flowchart. CCK8, Cell Counting Kit-8; LPS, lipopolysaccharide; PTZ, pentylenetetrazole.

no. bs-10442R) (all 1:500); anti-IL-6, (cat. no. bs-6309R), anti-IL-18 (cat. no. bs-4988R), anti-TNF- $\alpha$ (cat. no. bs-10802R), anti-active caspase-3 (cat. no. bsm-33199M), anti-Bax (cat. no. bs-0127R), anti-Bcl-2 (cat. no. bsm-52304R) and anti- $\beta$-actin (cat. no. bs-0061R) (all 1:1,000) (BIOSS); and anti- $\beta$-actin (cat. no. 66009-1-Ig; 1:5,000; ProteinTech Group, Inc.). Next, the membranes were washed with Tris-buffered saline (TBS) containing 5\%o Tween-20, and then incubated with goat anti-mouse IgG 800RD (cat. no. 926-32210; 1:5,000) or goat anti-rabbit IgG 680RD (cat. no. 925-68071; 1:5,000) (both BD Biosciences) at RT for $1.5 \mathrm{~h}$. Finally, Odyssey CLX software (9141-00; BD Biosciences) was used for imaging, and protein signals were quantified using ImageJ 8.0 software (National Institutes of Health). All experiments were conducted $\geq 3$ times.

Animals and treatment. C57BL/6J mice (male, $10 \pm 2$ weeks old; weight, $25 \pm 3 \mathrm{~g}$ ) were obtained from Beijing, China Fukang Biotechnology Co., Ltd. All protocols were approved by the Ethics Committee of Ningxia Medical University [approval no. SCXK(Ning)2019-203]. All mice were raised in the independent ventilation cage $\left(n=5\right.$ per cage) at $25 \pm 1^{\circ} \mathrm{C}$ with $50-60 \%$ relative humidity, free access to food and water, and 12-h light/dark cycles. All mice were anesthetized with $60 \mathrm{mg} / \mathrm{kg}$ sodium pentobarbital and decapitated for hippocampal tissue removal. Briefly, electrode implantation surgery was performed on the first day, and semaglutide was administered every other day for 37 days. At 8 days post-surgery, the mice received pentylenetetrazole (PTZ) every other day for 29 days. From the 38th day, a behavioral test was conducted for 7 days. Brain tissues were collected on the 45th day (Fig. 1B). The animals were randomized into five groups (20 per group), all of which were treated by intraperitoneal (i.p.) injection. In the control group (1\% DMSO, i.p.), mice received 20 injections in total - 19 injections every other day for 37 days, and a final injection on the 45 th day. For the semaglutide group $[25 \mathrm{nM} / \mathrm{kg}$ semaglutide i.p. (29)], the administration schedule was the same as that for the control group. In the PTZ group [37 mg/kg
PTZ, i.p. (1)], the mice received a total of 16 injections - one injection every other day from the 9 th to the 37 th day for a total of 15 injections, and a final injection on the 45th day. For the low-dose semaglutide group [37 mg/kg PTZ + $10 \mathrm{nM} / \mathrm{kg}$ semaglutide, i.p. (27)], the semaglutide administration schedule was the same as that for the control group, and the PTZ administration schedule was the same as that for the PTZ group, $30 \mathrm{~min}$ after each injection of semaglutide. For the high-dose semaglutide group $(37 \mathrm{mg} / \mathrm{kg}$ PTZ $+25 \mathrm{nM} / \mathrm{kg}$ semaglutide, i.p.), the administration schedule was the same as that for the low-dose semaglutide group.

Electrocorticography (ECoG). Intracranial electrodes were implanted 8 days before PTZ injection. Briefly, after the mice were anaesthetized with $60 \mathrm{mg} / \mathrm{kg}$ sodium pentobarbital, a stereotactic device was used to implant electrodes into the cerebral cortex on both sides of the bregma. The specific method for implanting the intracranial electrodes was the same as that used in our previous study (25). On the 45th day, seizure severity was assessed, and ECoG was recorded using an information integrated biological signal acquisition and processing system (BL-420 N; Techman).

Kindling model protocol. Mice were administered PTZ (37 mg/kg) every other day for 29 days. Seizure severity was evaluated using the modified Racine scale as follows: Stage one, mouse and facial twitching; stage two, nodding and clonus; stage three, mild unilateral or bilateral limb twitch; stage four, bilateral forelimb vibration accompanied by standing; stage five, generalized convulsions accompanied by falling; stage six, tonic convulsions with hindlimb extension; and stage seven, death. Three consecutive 4- or higher-stage convulsions indicated successful kindling (34).

\section{Behavioral assessment}

Modified novel object recognition (NOR). The NOR assessment evaluates the ability of the mouse to recognize a novel object under a specific condition, using the Smart 3.0 behavior video recording and analysis system (Panlab). The modified NOR test consists of the habituation phase, training phase and test phase. Each mouse was tested and evaluated in a sound-attenuated room. During the habituation phase, each mouse was adapted for 3 min before training. During the training phase with similar objects, a single mouse was placed in a closed square-shaped plane (arena, 55x55x55 cm) containing two plastic objects of the same shape (square, $7 \times 7 \times 7 \mathrm{~cm}$ ) for $6 \mathrm{~min}$. The test phase followed the training phase, and was conducted with different objects; the mouse was returned to the arena, one object was replaced with a novel plastic object (cylindrical shape; radius, $3.5 \mathrm{~cm}$; and height, $7 \mathrm{~cm}$ ) and the mouse was placed into the square for testing for $6 \mathrm{~min}$ at $20 \mathrm{~min}$ intervals. The arena and objects were cleaned between the two tests, completely eliminating olfactory cues. The discriminant index (DI) was defined as the time spent by the mouse exploring the new object as a percentage of the memory retention period. The total time was calculated as the time that the mouse spent exploring both objects. A DI of $50 \%$ is equal to the random chance percentage, and a higher DI indicates that the mouse prefers a certain object, indicating that it can remember similar objects and locations. 
Shuttle box active avoidance test. These trials were performed in a two-way shuttle box (MED-APAP-D1R; MED Associates, Inc.) consisting of two compartments accessible to each other by a guillotine door. Each experimental session included 30 trials and was repeated on five consecutive days. Each experiment included conditioned stimulation (simultaneous presentation of light and sound, $5 \mathrm{sec}$ ) and unconditioned stimulation (electric shock, $0.3 \mathrm{~mA}, 50 \mathrm{~Hz}, 10 \mathrm{sec}$ ) delivered via stainless steel rods on the floor of the apparatus. When the mouse walked into the other side of the shuttle box, the stimulus was immediately turned off. The number of total active avoidance responses (AARs) was recorded with Med-PC (version 5.1; MED Associates, Inc.) software for evaluation. Mice were tested on the 5th day.

Passive avoidance test. These trials were performed in a one-way shuttle box (MED-APAP-D1R; MED Associates, Inc.) with two equal-sized compartments (darkened/lit apparatus) separated by a guillotine door. The memory test was performed over 2 days. On the first day, a mouse was released into the lit cage and moved freely around for $5 \mathrm{~min}$ for habituation to the compartment. Then, the mouse was placed in the lit cage again, and the door opened; when the mouse entered the dark cage, the door was closed, and the grid floor delivered a mild electrical shock $(0.3 \mathrm{~mA}$ for $3 \mathrm{sec})$. After $120 \mathrm{sec}$, the animal was removed from the dark compartment and placed in the lit compartment again until it remained in the lit compartment for $120 \mathrm{sec}$, at which point the experiment was terminated. The number of electric shocks was recorded; $24 \mathrm{~h}$ later, passive avoidance learning was evaluated using the shuttle box, the latency time was recorded, and $120 \mathrm{sec}$ was set as the maximum latency time for each mouse.

Morris water maze (MWM) assessment. The MWM (WMT-100S; Techman Software) was performed in a pool with a diameter of $80 \mathrm{~cm}$ and a height of $50 \mathrm{~cm}$, filled with $23 \pm 2^{\circ} \mathrm{C}$ water, and a white circular platform with a diameter of $5 \mathrm{~cm}$ that allows the mouse to escape the water. The experimental method was as previously described (1). Briefly, the pool was divided into four quadrants, and the circular platform was placed in the first quadrant (target quadrant). Several distal cues were set around the pool and were maintained in the same positions throughout all the tests. The MWM assessment consisted of three parts: i) A visible circular platform trial; ii) a navigation trail; and iii) a spatial probe trail. The visual platform trial was first performed to assess the vision and motor function of each mouse, and the platform was placed $1.5 \mathrm{~cm}$ under the surface of the water. The mice were tested for 5 days, the four quadrants were tested each day, with a test interval of $15 \mathrm{~min}$. On the sixth day, the spatial probe test began. Mice were placed in the third quadrant and allowed to swim for $60 \mathrm{sec}$. The swimming tracking data were recorded.

Reverse transcription-quantitative $(R T-q) P C R$. Total RNA was extracted from the hippocampal tissues using an RNA simple Total RNA kit (Tiangen Biotech Co., Ltd.), and the FastKing gDNA Dispelling RT SuperMix kit (Tiangen Biotech Co., Ltd.) was used to synthesize cDNA. RT-qPCR was performed using the FastFire qPCR PreMix (SYBR-Green; Tiangen Biotech Co., Ltd.) according the manufacturer's protocol. The following primers were used for qPCR: NLRP3 forward, 5'-GGAGGAA GAAGAAGAGAGGAGAGGAG-3' and reverse, 5'-CTTGAGA AGAGACCACGGCAGAAG-3'; ASC forward, 5'-ACAATGA CTGTGCTTAGAGACA-3 and reverse, 5'-CACAGCTCCAGA CTCTTCTTTA-3'; caspase-1 p20 forward, 5'-TGAATACAAC CACTCGTACACGTCTTG-3' and reverse, 5'-CCAGATCCT CCAGCAGCAACTTC-3'; and GAPDH (mouse endogenous reference gene primers; cat. no. B661304). All primers were purchased from Shenggong Bioengineering Co., Ltd. The reaction conditions were in accordance with the manufacturer's instructions: Denaturation at $95^{\circ}$ for $15 \mathrm{~min}$, followed by 40 cycles of denaturation at $95^{\circ}$ for $10 \mathrm{sec}$, annealing at $61.5^{\circ}$ for $20 \mathrm{sec}$ and extension at $72^{\circ}$ for 30 sec; iQ ${ }^{\mathrm{TM}} 5$ software (Bio-Rad Laboratories, Inc.) was used to conduct PCR amplification. The RNA expression levels were evaluated using the $2^{-\Delta \Delta \mathrm{Cq}}$ method (35). PRISM 8 software (GraphPad Software, Inc.) was used to evaluate the relative RNA level. All experiments were carried out $>3$ times.

Nissl staining. After the mice were anaesthetized, they were intracardially perfused with $4 \%$ paraformaldehyde. Then, the brain tissue was collected and soaked in fixative for $12 \mathrm{~h}$ at RT. Specimens were dehydrated and immersed in paraffin, and were then sliced to a thickness of $5 \mu \mathrm{m}$ at RT for later use. The paraffin sections were sequentially incubated in xylene solution A and xylene solution B for $19 \mathrm{~min}$, followed by incubation in anhydrous ethanol solution A, anhydrous ethanol solution B, and $75 \%$ alcohol for $6 \mathrm{~min}$ at RT. The sections were then washed with flowing water.

Mouse tissue sections were placed in staining solution for 6 min and slightly differentiated with $1 \%$ glacial acetic acid at RT. The reaction was terminated by washing with tap water. The degree of differentiation was controlled by evaluation under a fluorescent microscope. For transparent mounting, the slices were incubated in fresh xylene for $6 \mathrm{~min}$ and mounted with neutral gum at RT. Sections were imaged with Pannoramic DESK, p-MIDI, p250 (3D HISTECHE, Ltd.), and analyzed using ImageJ 8.0 software.

Immunofluorescence staining. The method used to prepare the tissue sections was the same as that used for Nissl staining. Subsequently, ethylenediaminetetraacetic acid ( $\mathrm{pH} \mathrm{8.0)}$ was used for antigen retrieval at RT, after which the sections were washed with PBS ( $\mathrm{pH}$ 7.4). Subsequently, the sections were incubated in an autofluorescence quencher agent reagent (cat. no. G1221; Wuhan Servicebio Technology Co., Ltd.) for $5 \mathrm{~min}$ at RT, rinsed with running water for $10 \mathrm{~min}$, and then incubated with $5 \%$ BSA for $30 \mathrm{~min}$ at RT to block nonspecific reactions. Next, the sections were incubated with anti-active caspase-3, anti-Bax, anti-Bcl-2, anti-NLRP3, anti-ASC, anti-caspase-1 p20, anti-NeuN (cat. no. bs-10394R; BIOSS) and anti-Iba-1 [ab283319, Abcam (all 1:500)] antibodies for $15 \mathrm{~h}$ at $4^{\circ} \mathrm{C}$. Then, the sections were washed 3 times with PBS and subsequently incubated with the associated secondary antibodies (all 1:1,000) for $90 \mathrm{~min}$ at RT. Finally, the sections were covered with a sealer containing DAPI, imaged using Pannoramic DESK, p-MIDI, p250 and analyzed using ImageJ 8.0 software.

Statistical analysis. PRISM 8 software was used for statistical analysis. The results are expressed as the mean \pm standard deviation. All experiments were performed at least three times. Differences between two factors was compared using 
A

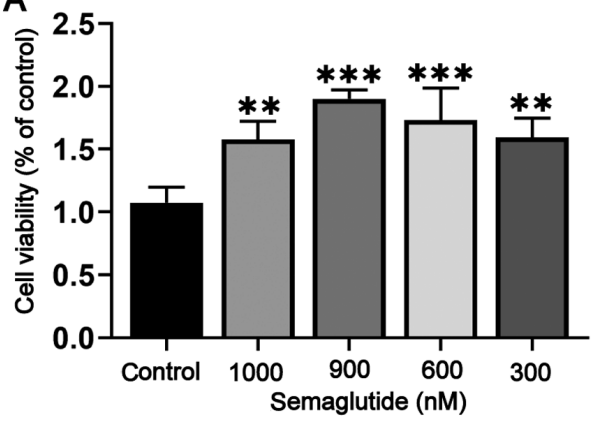

C

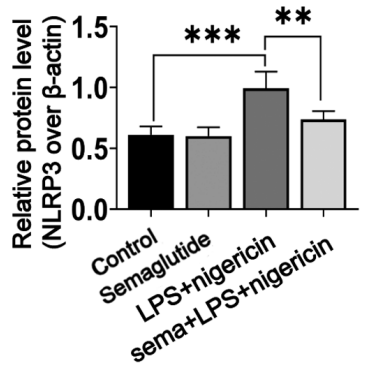

G

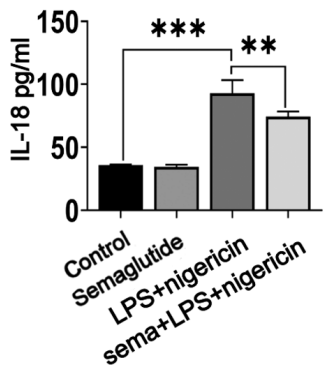

D

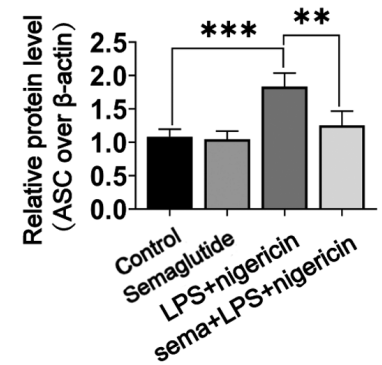

H

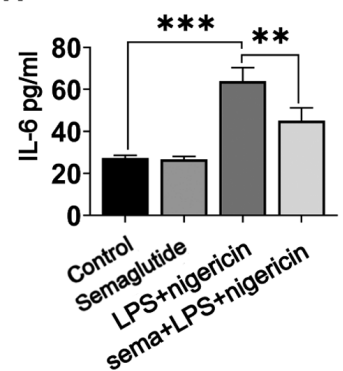

B

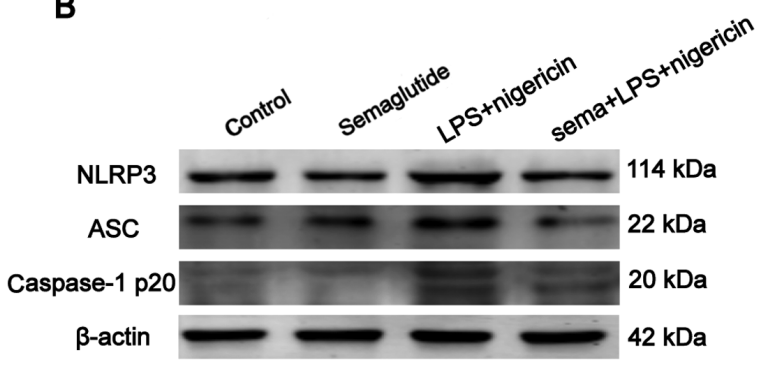

E

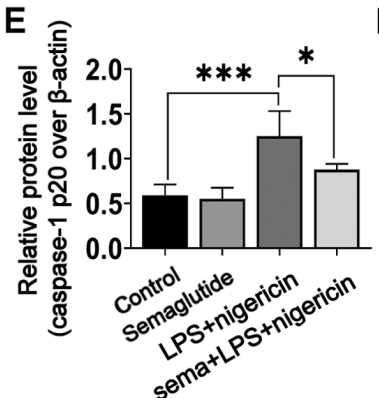

$\mathbf{F}$

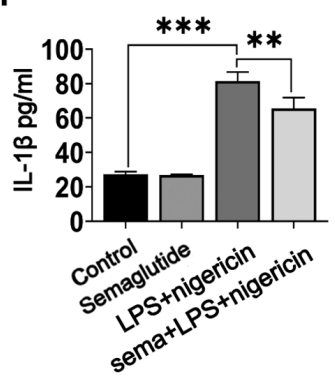

$\mathbf{J}$
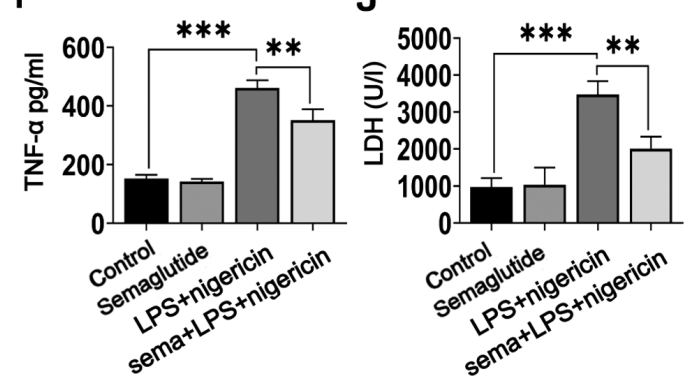

Figure 2. Effect of semaglutide treatment on BV2 cells mediated by LPS and nigericin. (A) Proliferation of BV2 cells treated with semaglutide at different concentrations $(300,600,900$ and $1,000 \mathrm{nM}$ ) was assessed using Cell Counting Kit- 8 analysis followed by one-way ANOVA. (B) Representative WB images in different BV2 cell groups. (C-E) Statistical results of WB band intensities (Student's t-test). (F-I) Statistical results of IL-1 $\beta$, IL-18, IL-6 and TNF- $\alpha$ secretion, as assessed by ELISA (Student's t-test). (J) Semaglutide inhibited LPS- and nigericin-mediated LDH release in BV2 cells (Student's t-test). Data are presented as the means $\pm \mathrm{SD} .{ }^{*} \mathrm{P}<0.05,{ }^{* *} \mathrm{P}<0.01$ and ${ }^{* * * *} \mathrm{P}<0.001$. N $>3$. LPS, lipopolysaccharide; WB, western blotting/blot; LDH, lactate dehydrogenase; NLRP3, NLR family pyrin domain containing 3 ; ASC, apoptosis-associated speck-like protein.

unpaired Student's t-test, and multivariate differences were compared by one-way or repeated measures two-way ANOVA followed by Tukey's post hoc test. $\mathrm{P}<0.05$ was considered to indicate a statistically significant difference.

\section{Results}

Semaglutide attenuates LPS- and nigericin-induced inflammatory responses by blocking the NLRP3 inflammasome in vitro. Seizures promote brain inflammation and activate microglia; activated microglia in turn exacerbate inflammation, which further aggravates epilepsy (36). Therefore, in the present study, the anti-inflammatory effect of semaglutide was evaluated using BV2 cells. The most appropriate concentration of semaglutide for the treatment of BV2 cells was determined using the CCK-8. The findings suggested that semaglutide did not exhibit cytotoxicity and minimally impacted cell viability at either low or high doses. Therefore, $900 \mathrm{nM}$ was selected as the optimal semaglutide concentration to assess cell viability for subsequent experimentation (Fig. 2A; P<0.001).

WB analysis was used to evaluate the expression of the NLRP3 inflammasome in BV2 cells (Fig. 2B). NLRP3 inflammasome expression was not significantly different between the semaglutide group and the controls (Fig. 2C-E), but was increased in the LPS + nigericin group $(\mathrm{P}<0.001)$. Indeed, semaglutide pretreatment followed by LPS + nigericin significantly reduced NLRP3 inflammasome activation $(\mathrm{P}<0.05)$. In addition, the ELISA results showed that the levels of IL-1 $\beta$, IL-18, IL- 6 and TNF- $\alpha$ were significantly higher in the LPS + nigericin group than in the control groups (Fig. 2F-I; $\mathrm{P}<0.001)$. Semaglutide pretreatment significantly suppressed the secretion of inflammatory cytokines mediated by LPS + nigericin $(\mathrm{P}<0.01)$, but semaglutide alone did not affect these levels. These data revealed that semaglutide attenuated LPS + nigericin-induced NLRP3 inflammasome activation in BV2 cells.

Semaglutide inhibits LPS- and nigericin-mediated $L D H$ release in $B V 2$ cells. Pyroptosis is a type of programmed necrosis that is involved in inflammatory cell death. NLRP3 inflammasome activation produces active caspase- 1 and promotes the release of the inflammatory factor IL- $1 \beta$ from cells, which is an important mechanism leading to pyroptosis. LDH release is often measured to assess the level of 
A

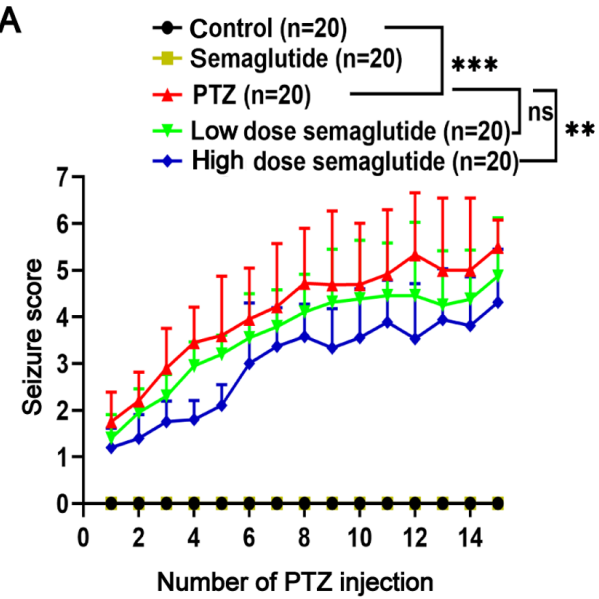

B

Control

Semaglutide

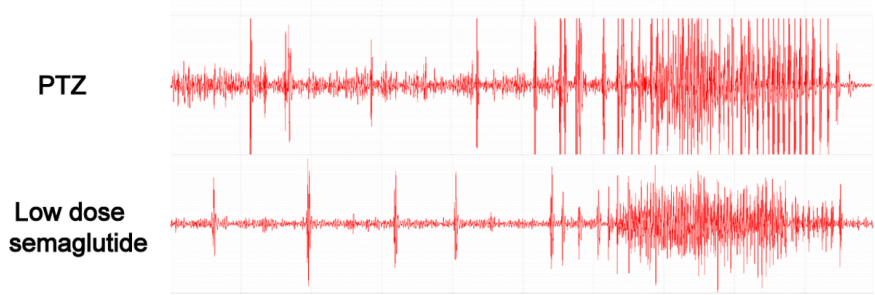

High dose semaglutide

D

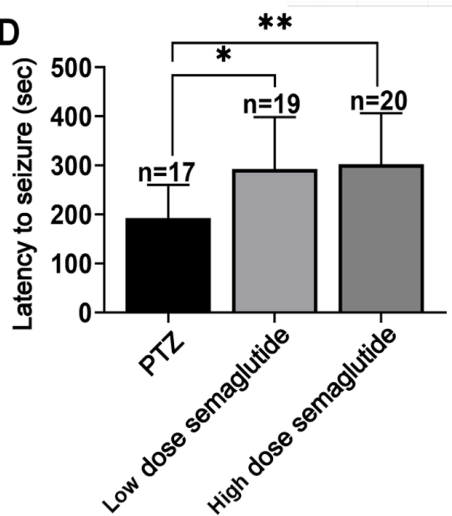

E

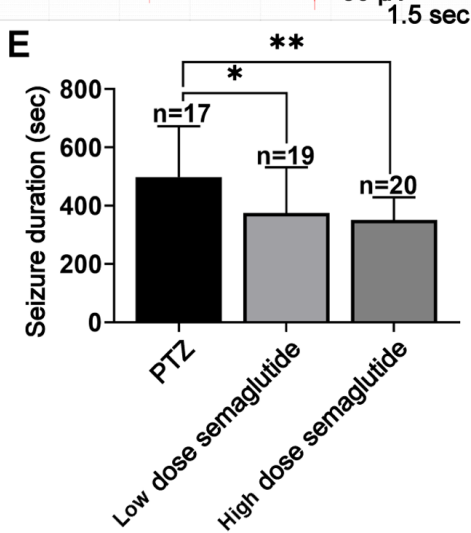

C

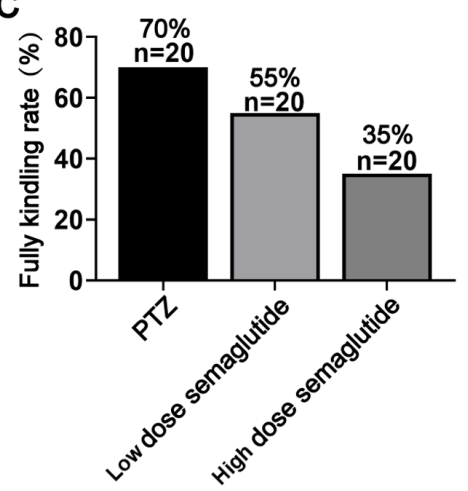

Figure 3. Semaglutide reduces PTZ-induced seizure severity. (A) Statistical results showed that semaglutide doses of 10 and $25 \mathrm{nM} / \mathrm{kg}$ decreased the modified Racine score (two-way ANOVA). (B) Representative electrocorticography results in each group of mice. (C) Both 10 and $25 \mathrm{nM} / \mathrm{kg}$ semaglutide decreased the rate of complete kindling. (D) Both the 10 and $25 \mathrm{nM} / \mathrm{kg}$ semaglutide increased the latency to generalized seizures (one-way ANOVA). (E) Both the 10 and $25 \mathrm{nmol} / \mathrm{kg}$ semaglutide alleviated the duration of generalized seizures (one-way ANOVA). Data are presented as the means $\pm \mathrm{SD}$. ${ }^{*} \mathrm{P}<0.05,{ }^{* * *} \mathrm{P}<0.01$ and ${ }^{* * * *} \mathrm{P}<0.001$. N $\geq 17$. PTZ, pentylenetetrazole.

pyroptosis after inflammasome activation (37). Semaglutide was confirmed to inhibit the release of caspase- 1 p20 and IL-1 $\beta$ after LPS- and nigericin-mediated NLRP3 inflammasome activation. Therefore, the potential effects of semaglutide on BV2 cell pyroptosis were further investigated. Analysis of cell supernatants showed that stimulation with LPS and nigericin promoted the release of LDH (Fig. $2 \mathrm{~J} ; \mathrm{P}<0.001$ ) and that semaglutide inhibited LDH release mediated by LPS + nigericin $(\mathrm{P}<0.01)$, indicating that semaglutide can inhibit LPS + nigericin-mediated pyroptosis.

Semaglutide reduces seizure severity in PTZ-kindled mice. Semaglutide exerted anti-inflammatory and anti-pyroptotic effects on LPS- and nigericin-activated BV2 cells. Therefore, the potential effects of semaglutide on PTZ-kindled mice were also investigated. The modified Racine score and rate of complete kindling were recorded every other day for 29 days (15 injections). After the last (16th) injection on the 45th day, the latency to seizure, seizure duration and ECoG were recorded. Pretreatment with semaglutide (10 and $25 \mathrm{nM} / \mathrm{kg}$, i.p.) decreased the seizure score (Fig. 3A; $\mathrm{P}>0.05$ and $\mathrm{P}<0.01$, respectively) and reduced the rate of complete kindling during 15 injections of PTZ (55 and 35\%, respectively) compared with the PTZ group (70\%) (Fig. 3A and C). Both doses of semaglutide (10 and $25 \mathrm{nM} / \mathrm{kg}$; i.p.) partially increased the latency to generalized seizures (Fig. 3D; $\mathrm{P}<0.05$ and $\mathrm{P}<0.01$, respectively) and shortened the seizure duration (Fig. 3E; $\mathrm{P}<0.05$ and $\mathrm{P}<0.01$, respectively). Furthermore, the ECoG results showed a lower frequency and amplitude of spike-wave discharges in the low- and high-dose semaglutide groups than in the PTZ group (Fig. 3B).

Semaglutide affects learning and memory in PTZ-kindled mice

NOR test. No significant differences were observed between the two similar objects during the test and training phases. The results of the NOR test revealed no significant differences in the DI between the semaglutide-treated and control mice (Fig. 4A). Conversely, the PTZ mice had a lower DI than the controls $(\mathrm{P}<0.001)$. In addition, mice treated with either dose of semaglutide ( 10 or $25 \mathrm{nM} / \mathrm{kg}$ ) spent more time in the area of the new object and had a higher DI than the PTZ mice ( $\mathrm{P}>0.05$ and $\mathrm{P}<0.01$, respectively).

Shuttle box active avoidance test. The number of AARs increased gradually over the 5 days of training in all groups. However, the AAR time was lower in the PTZ mice than in the control mice (Fig. 4B; $\mathrm{P}<0.0001$ ). In both semaglutide groups (low- and high-dose), the number of AARs was increased 
A

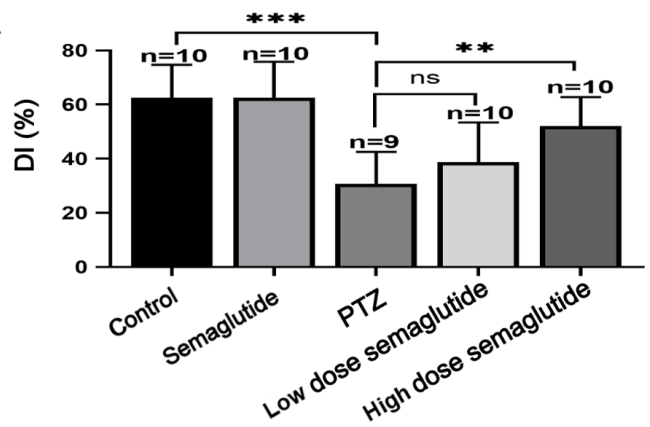

C

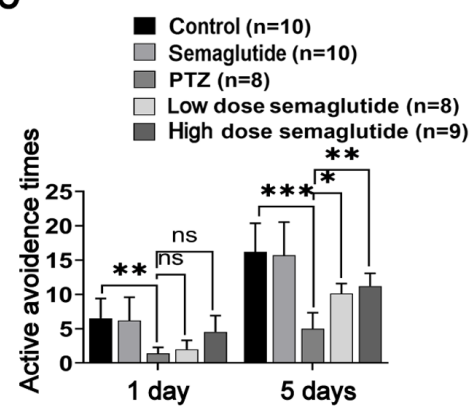

F

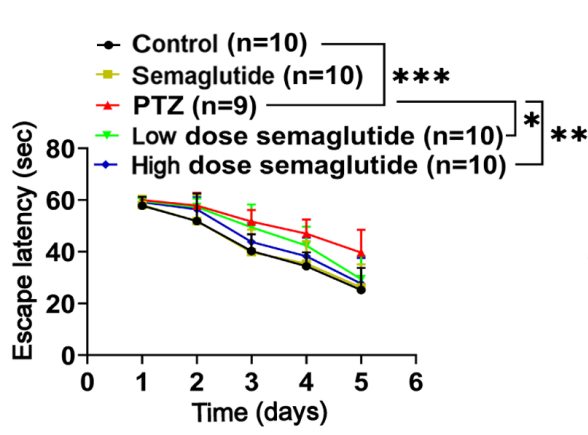

B

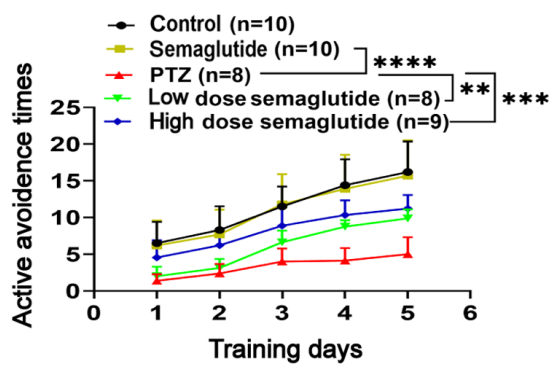

D

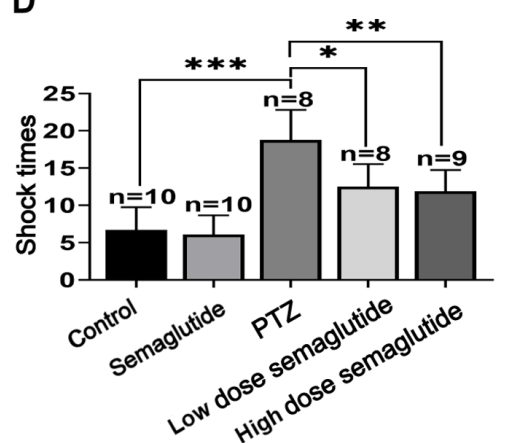

E

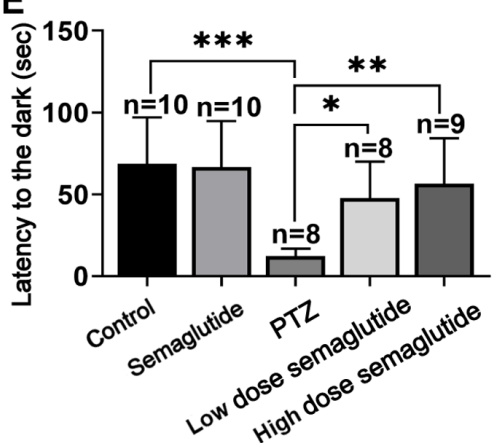

H
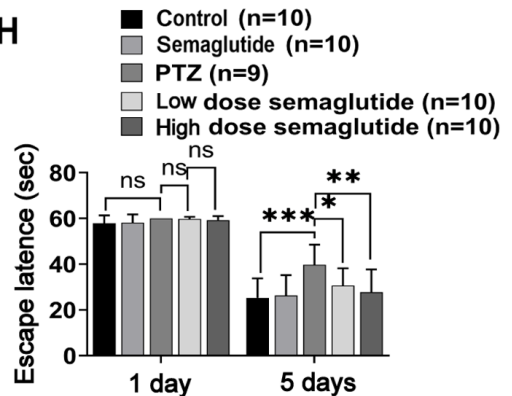

G

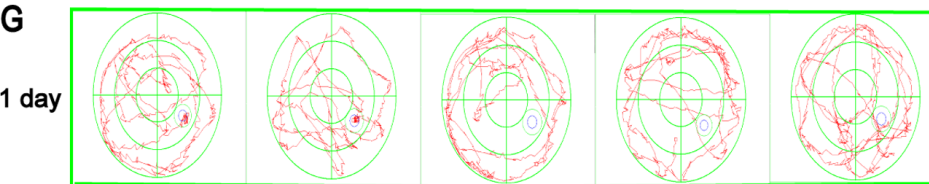

5 days

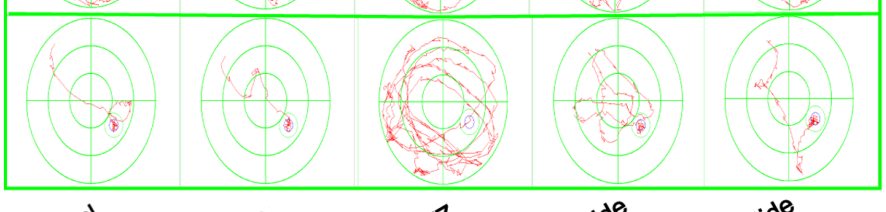

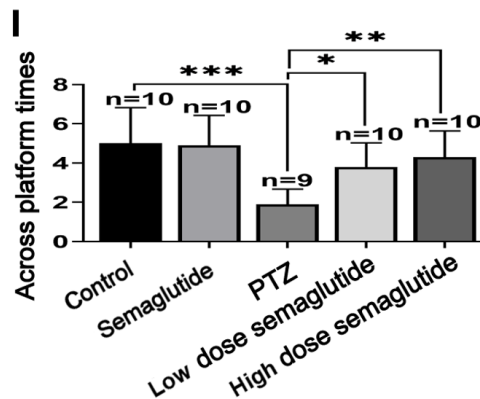
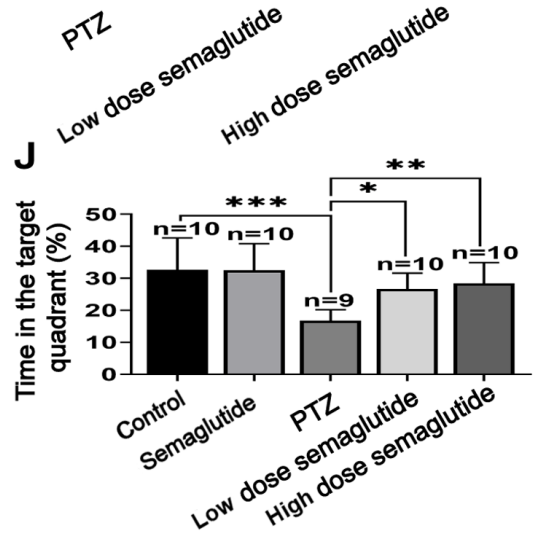

Figure 4. Assessment of behavioral parameters. (A) Statistical results showed that the DI of each group in the novel object recognition test. Both 10 and $25 \mathrm{nM} / \mathrm{kg}$ semaglutide increased the DI in comparison to PTZ group (one-way ANOVA). (B and C) Statistical results showed that both the 10 and $25 \mathrm{nM} / \mathrm{kg}$ semaglutide increased the active avoidance time in the active avoidance test in comparison with PTZ group (two-way repeated ANOVA). (D and E) Statistical results showed that both the 10 and $25 \mathrm{nM} / \mathrm{kg}$ doses of semaglutide reduced the shock time and increased the latency to enter the dark cage in the passive avoidance test in comparison with PTZ group (one-way ANOVA). (F-J) Statistical results for each group of mice in the Morris water maze test. (F) Statistical results showed that both doses of semaglutide $(10$ and $25 \mathrm{nM} / \mathrm{kg}$ ) reduced the escape latency in comparison with PTZ group (two-way repeated ANOVA test). $(\mathrm{G}$ and $\mathrm{H})$ Representative tracks and statistical results showed that both the 10 and $25 \mathrm{nM} / \mathrm{kg}$ doses of semaglutide reduced the escape latency on the first and fifth days of training in comparison with PTZ group (two-way ANOVA). (I and J) Statistical results showed that the time spent in the target district and the number of crossed times were increased after treatment with semaglutide at doses of 10 and $25 \mathrm{nM} / \mathrm{kg}$ in comparison with PTZ group (one-way ANOVA). Data are presented as the means \pm SD. ${ }^{*} \mathrm{P}<0.05,{ }^{* *} \mathrm{P}<0.01,{ }^{* * *} \mathrm{P}<0.001$ and ${ }^{* * * * *} \mathrm{P}<0.0001$. N $\geq 8$. DI, discriminant index; PTZ, pentylenetetrazole.

compared with that in the PTZ mice $(\mathrm{P}<0.01$ and $\mathrm{P}<0.001$, respectively). The number of AARs in the semaglutide mice and the controls was not significantly different. Moreover, on the first day, the number of AARs was not significantly different in any group. However, on the fifth day, the success rate on the active avoidance test was increased in both semaglutide groups (low- and high-dose) with respect to the PTZ mice (Fig. 4C; $\mathrm{P}<0.05$ and $\mathrm{P}<0.01$, respectively).

Passive avoidance test. Before receiving a shock, the latency time to enter the dark cage was not significantly different between any of the groups. In addition, in the training test, the number of training sessions did not differ between 
the semaglutide-treated mice and controls (Fig. 4D). However, the number of training sessions was significantly higher in the PTZ-treated mice compared with the controls $(\mathrm{P}<0.001)$. In both semaglutide groups (low- and high-dose), the number of training sessions was significantly decreased with respect to that in the PTZ mice $(\mathrm{P}<0.05$ and $\mathrm{P}<0.01$, respectively). The test trial was carried out 1 day later in the training trial. The delayed period to enter the dark cage did not differ between the semaglutide and control mice (Fig. 4E). Notably, the delay to enter the dark cage was shorter in the PTZ mice than in the controls $(\mathrm{P}<0.001)$ and longer in mice in both semaglutide groups (low- and high-dose) than in the PTZ mice $(\mathrm{P}<0.05$ and $\mathrm{P}<0.01$, respectively).

MWM test. In the visible platform trial, the escape latency did not significantly differ between the groups. In the positioning cruise test, the escape latency gradually decreased in all groups (Fig. 4F-G). For the semaglutide mice, the escape latency did not differ with respect to the controls. However, in the PTZ mice, the escape latency was significantly longer with respect to the control mice $(\mathrm{P}<0.001)$. The escape latency was partially reduced by both concentrations of semaglutide (low- and high-dose) with respect to the PTZ alone $(\mathrm{P}<0.05$ and $\mathrm{P}<0.01$, respectively). On the first training day, there was no significant difference in escape latency between any of the groups (Fig. 4H). On the last training day (day 5), the escape latency was longer in the PTZ mice than in the controls $(\mathrm{P}<0.001)$. In both semaglutide groups (low- and high-dose), the escape latency was partially reduced compared with that in the PTZ mice $(\mathrm{P}<0.05$ and $\mathrm{P}<0.01$, respectively).

In the spatial probe trial, the time spent in the target quadrant and the number of crossed times were not significantly different between the semaglutide mice and the controls (Fig. 4I-J). However, the number of crossings in the PTZ group was significantly lower with respect to the controls over a 60 -sec period $(\mathrm{P}<0.001)$. Both doses of semaglutide $(10$ and $25 \mathrm{nM} / \mathrm{kg})$ partially increased the number of crossings compared with the $\mathrm{PTZ}$-alone group $(\mathrm{P}<0.05$ and $\mathrm{P}<0.01$, respectively). Collectively, these data suggested that semaglutide minimized PTZ- induced cognitive impairment in mice.

Semaglutide decreases hippocampal neuron apoptosis in PTZ kindled-mice. Cognitive dysfunctions are closely related to hippocampal structure (20); therefore, the effect of semaglutide on hippocampal neuronal apoptosis was further evaluated.

First, Nissl staining was used for histological examination. The number of hippocampal neurons was similar between the semaglutide mice and the controls, and there were no obvious abnormalities in cell morphology or structure in the semaglutide mice (Fig. 5A-C). However, the number of the CA1 and CA3 hippocampal neurons were reduced in the PTZ mice $(\mathrm{P}<0.01$ and $\mathrm{P}<0.001$, respectively), and evaluation of neuronal morphology and structure revealed nuclear pyknosis and fragmentation relative to the controls (Fig. 5A). The number of the CA1 hippocampal neurons was increased in both semaglutide groups (low- and high-dose), and reduced nuclear pyknosis and fragmentation were observed relative to those in the PTZ mice (Fig. 5A and $\mathrm{B} ; \mathrm{P}>0.05$ and $\mathrm{P}<0.05$ ). The number of $\mathrm{CA} 3$ hippocampal neurons was increased and nuclear pyknosis and fragmentation were decreased in these mice relative to the PTZ mice (Fig. 5A and $\mathrm{C} ; \mathrm{P}<0.05$ and $\mathrm{P}<0.001$ ).
NeuN is located in the nucleus and cytoplasm; DAPI is located in the nucleus. NeuN has a larger fluorescence range, and the overall color after merging is green. Double immunofluorescence staining for $\mathrm{NeuN}$ and Iba-1 showed no significant differences in the semaglutide mice with respect to the controls (Fig. 5D-F). However, the fluorescence intensity of NeuN was decreased, but the fluorescence intensity of Iba-1 was higher in the PTZ mice with respect to the controls $(\mathrm{P}<0.001)$. By contrast, the fluorescence intensity of NeuN was higher and the fluorescence intensity of Iba-1 was lower in mice in both semaglutide groups (low- and high-dose) with respect to the PTZ mice $(\mathrm{P}<0.01$ and $\mathrm{P}<0.001$, respectively).

In addition, active caspase-3, Bax, and Bcl-2 were detected in hippocampal tissues using immunofluorescence staining and WB analysis. There were no significant differences in the immunofluorescence intensities of active caspase-3, Bax and Bcl-2 between the semaglutide mice and the control mice (Fig. 6A-F). The immunofluorescence intensities of active caspase-3 and Bax were significantly higher (Fig. 6A-D; $\mathrm{P}<0.001$ ), while the immunofluorescence intensities of Bcl-2 was lower (Fig. 6E and F; P<0.001) in the PTZ mice than in the controls. However, the effects of PTZ were partially blocked by both semaglutide doses (low- and high-dose): the fluorescence intensities of active caspase-3 and Bax were lower and that of Bcl-2 was higher in the CA1 region in both semaglutide mouse groups than in $\mathrm{PTZ}$ mice $(\mathrm{P}<0.01$ and $\mathrm{P}<0.001)$. Moreover, both semaglutide doses (low- and high-dose) reduced the fluorescence intensities of active caspase- 3 and $\mathrm{Bax}(\mathrm{P}<0.01$ and $\mathrm{P}<0.001$, respectively) and increased the fluorescence intensity of Bcl-2 ( $\mathrm{P}<0.05$ and $\mathrm{P}<0.001$, respectively) with respect to the $\mathrm{PTZ}$ mice in the $\mathrm{CA} 3$ region.

Finally, the WB results were similar to the immunofluorescence results. With respect to the corresponding values in the controls, the protein level of active caspase- 3 was increased and the ratio of $\mathrm{Bcl}-2 / \mathrm{Bax}$ was decreased in the PTZ mice (Fig. 6G-I; P<0.001). However, the expression of active caspase-3 was partially decreased in both semaglutide groups (low- and high-dose) with respect to the PTZ mice (Fig. 6H; $\mathrm{P}<0.01$ and $\mathrm{P}<0.001$, respectively). The ratio of $\mathrm{Bcl}-2 / \mathrm{Bax}$ was also decreased in both semaglutide groups (low- and high-dose) with respect to the PTZ mice (Fig. 6I; $\mathrm{P}<0.05$ and $\mathrm{P}<0.01$, respectively). Injection of semaglutide alone did not significantly affect the levels of apoptosis-related proteins.

Semaglutide blocks NLRP3 inflammasome activation in PTZ kindled-mice. It was confirmed that semaglutide attenuated the LPS- and nigericin-mediated inflammatory response by blocking the effects of NLRP3 inflammasome activation in vitro. Semaglutide attenuated seizure severity, ameliorated cognitive impairment and decreased hippocampal neuronal apoptosis in PTZ-kindled mice. Therefore, the potential for semaglutide to block NLRP3 inflammasome activation was then investigated in vivo. The mRNA levels in the hippocampus were assessed by RT-qPCR, and the protein levels were evaluated by WB analysis and immunofluorescence staining. In the PTZ mice, the mRNA level of the NLRP3 inflammasome in the hippocampus was significantly higher with respect to those in the controls (Fig. 7A-C; $\mathrm{P}<0.001$ ), while the mRNA level of the NLRP3, ASC and caspase-1p20 were decreased by low-dose $(\mathrm{P}<0.01, \mathrm{P}<0.001$ and $\mathrm{P}<0.05$, 


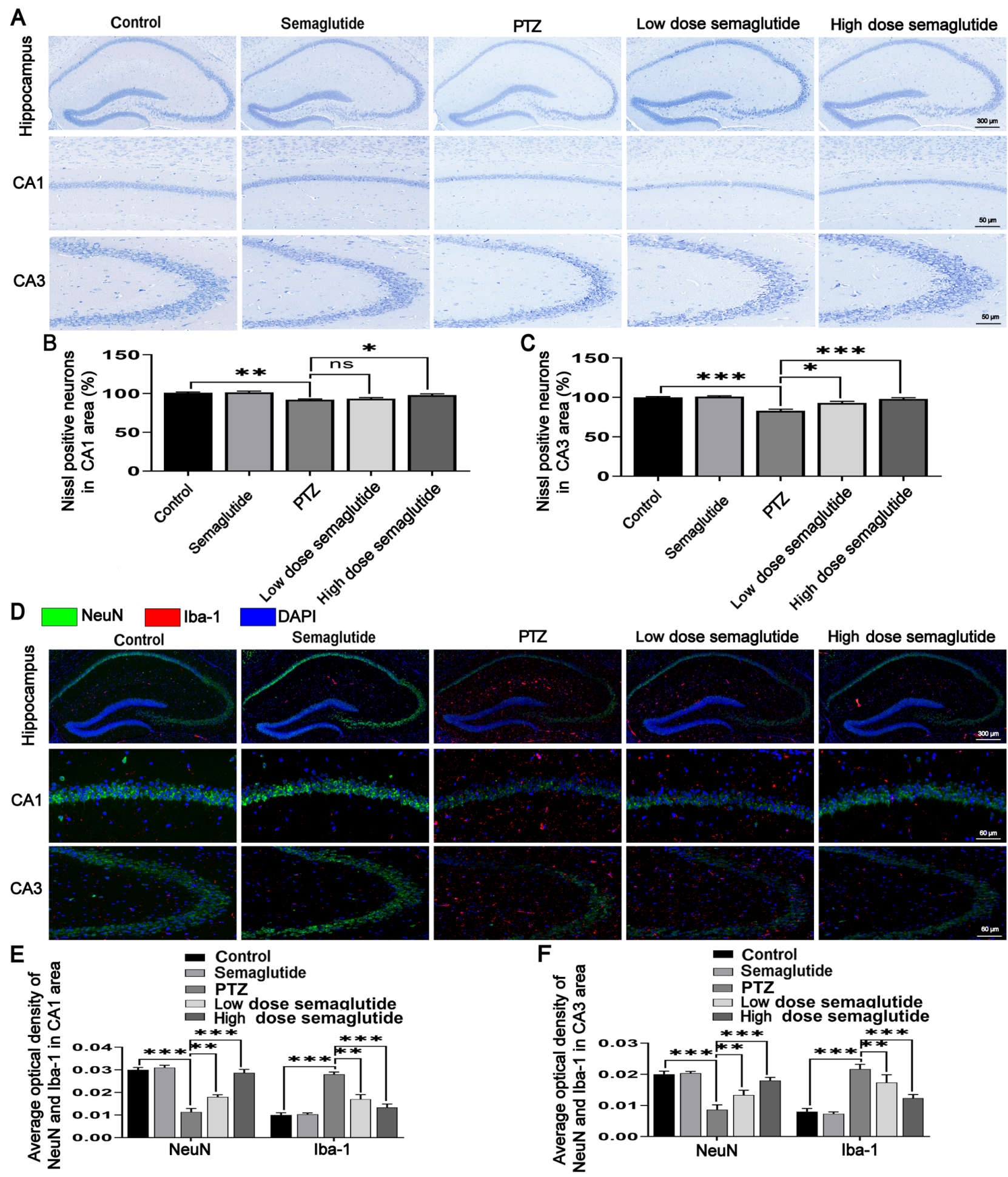

Figure 5. Results of Nissl staining and double immunofluorescence staining for NeuN and Iba-1 in the mouse hippocampus. (A) Representative images and (B and C) quantitative data for the number of Nissl-positive hippocampal neurons. Both 10 and $25 \mathrm{nM} / \mathrm{kg}$ semaglutide increased the numbers of Nissl-positive hippocampal neurons in the CA1 and CA3 areas (one-way ANOVA). (D) Representative immunofluorescence images and (E and F) densitometric analysis of NeuN and Iba-1 showing that both doses of semaglutide (enhanced the fluorescence intensity of Neun and decreased the fluorescence intensity of Iba-1 in the $\mathrm{CA} 1$ and CA3 areas (two-way ANOVA). Data are presented as the means $\pm \mathrm{SD}$. ${ }^{*} \mathrm{P}<0.05,{ }^{* * *} \mathrm{P}<0.01$ and ${ }^{* * * *} \mathrm{P}<0.001$. N $\geq 3$. $\mathrm{PTZ}$, pentylenetetrazole.

respectively) and high-dose (all $\mathrm{P}<0.001)$ semaglutide-treated mice compared with PTZ mice (Fig. 7A-C). However, semaglutide alone had no significant influence with respect to the controls.

The protein expression of the NLRP3 inflammasome was similar to its RNA level. NLRP3 inflammasome expression was higher in the PTZ mice with respect to the control mice (Fig. 7D-G; P<0.001). The protein levels of NLRP3, ASC and caspase-1 p20 were partially inhibited in low-dose $(\mathrm{P}<0.01, \mathrm{P}<0.05$ and $\mathrm{P}<0.01$, respectively) and high-dose semaglutide mice $(\mathrm{P}<0.001, \mathrm{P}<0.01$ and $\mathrm{P}<0.001$, respectively) with respect to the PTZ mice (Fig. 7E-G). However, semaglutide alone failed to influence the protein expression of the NLRP3 inflammasome with respect to that in the controls. 


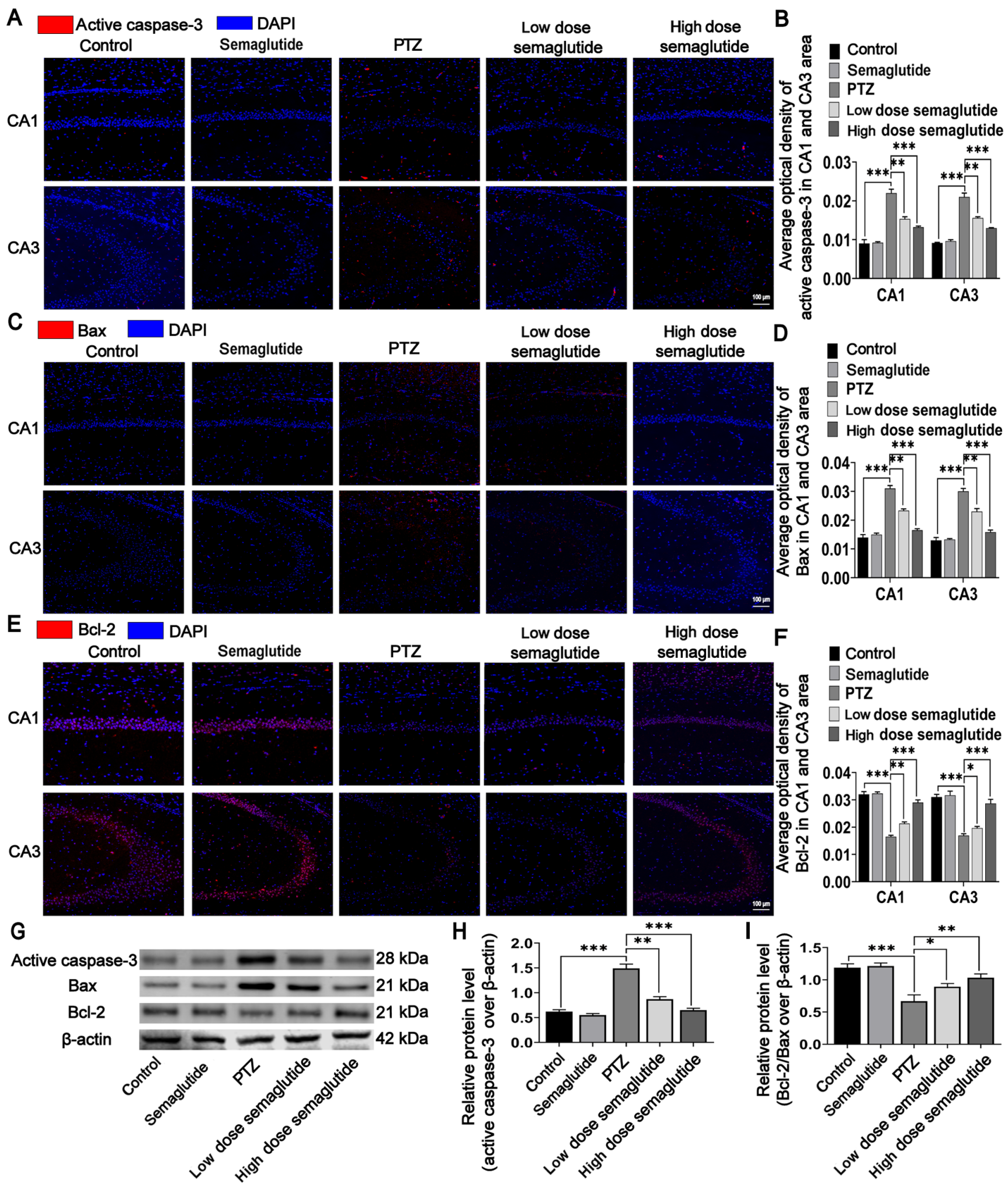

Figure 6. Immunofluorescence and WB analyses of apoptosis-related proteins in mouse hippocampal tissue. (A) Representative immunofluorescence images and (B) densitometric analysis of active caspase-3; both 10 and $25 \mathrm{nM} / \mathrm{kg}$ semaglutide decreased the fluorescence intensity of active caspase-3 in the CA1 and CA3 regions (one-way ANOVA). (C) Representative immunofluorescence images and (D) densitometric analysis of Bax showing that both doses of semaglutide decreased the fluorescence intensity of Bax in the CA1 and CA3 regions (one-way ANOVA). (E) Representative immunofluorescence images and (F) densitometric analysis of Bcl-2 showing that both 10 and $25 \mathrm{nM} / \mathrm{kg}$ semaglutide increased the fluorescence intensity of Bcl-2 in the CA1 and CA3 regions (one-way ANOVA). (G) Representative WB images of active caspase-3, Bax and Bcl-2 in the different groups. (H and I) Statistical results of the immunoblot analysis showing that 10 and $25 \mathrm{nM} / \mathrm{kg}$ semaglutide reduced the band intensity of active caspase-3 (one-way ANOVA) and increased the Bcl-2/Bax ratio (two-way ANOVA). Data are presented as the means $\pm \mathrm{SD}$. ${ }^{*} \mathrm{P}<0.05,{ }^{* * *} \mathrm{P}<0.01$ and ${ }^{* * * *} \mathrm{P}<0.001$. N $\geq 3$. WB , western blot/blotting; PTZ, pentylenetetrazole.

The immunofluorescence results were similar to those of WB and RT-qPCR. The immunofluorescence intensity of
NLRP3 successively decreased from the PTZ mice (Fig. 7H-I; $\mathrm{P}<0.001)$ to the low-dose semaglutide mice $(\mathrm{P}<0.05)$ to the 


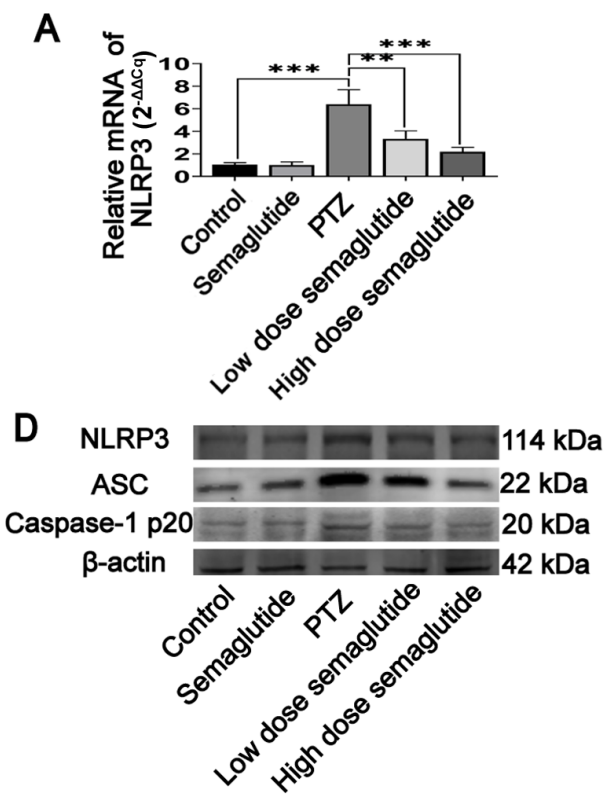

B

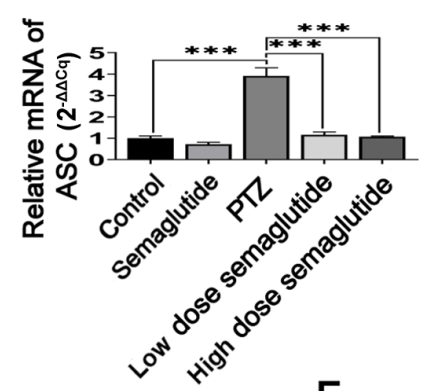

C

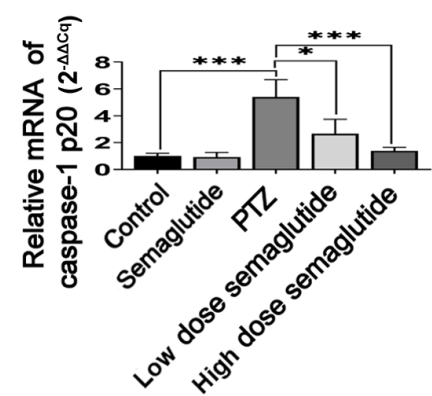

E

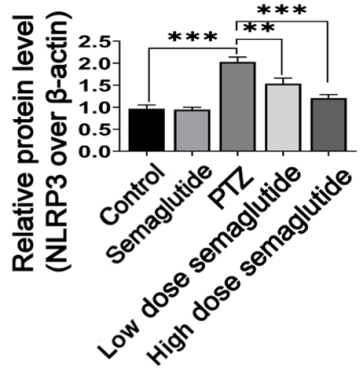

$F$

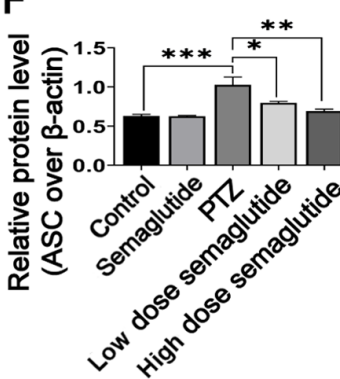

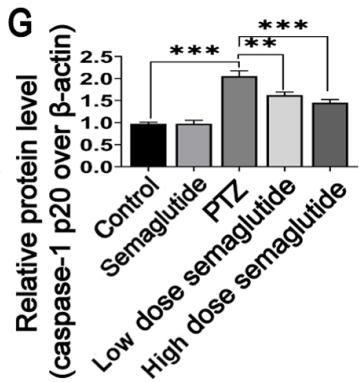
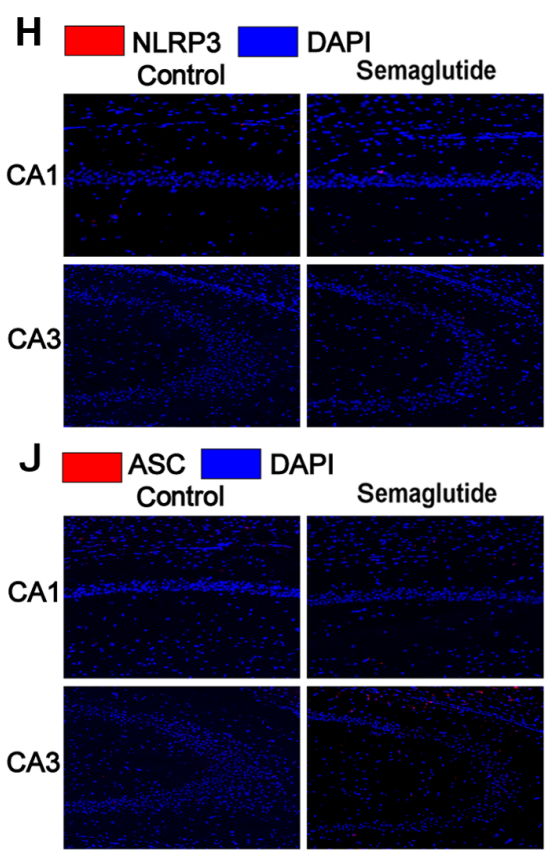

Semaglutide
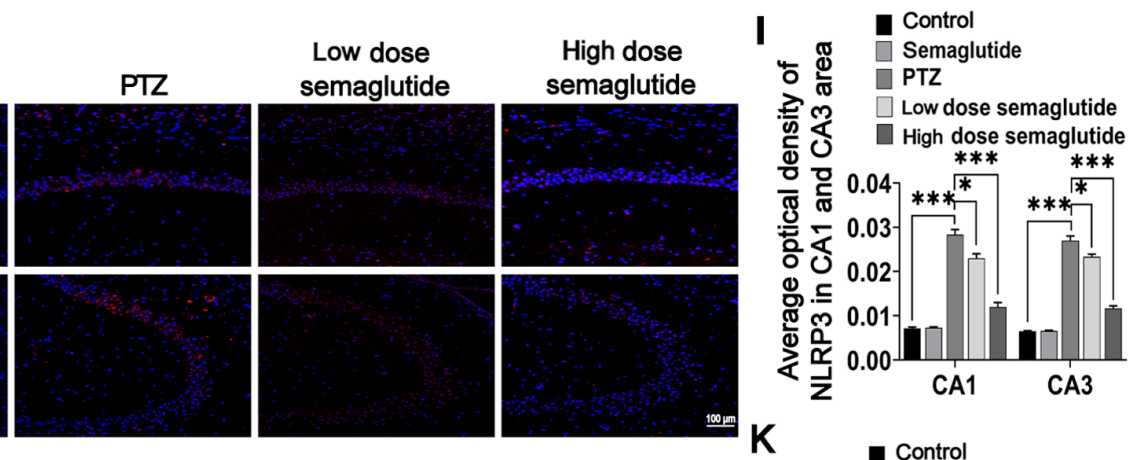

$\mathrm{K}$
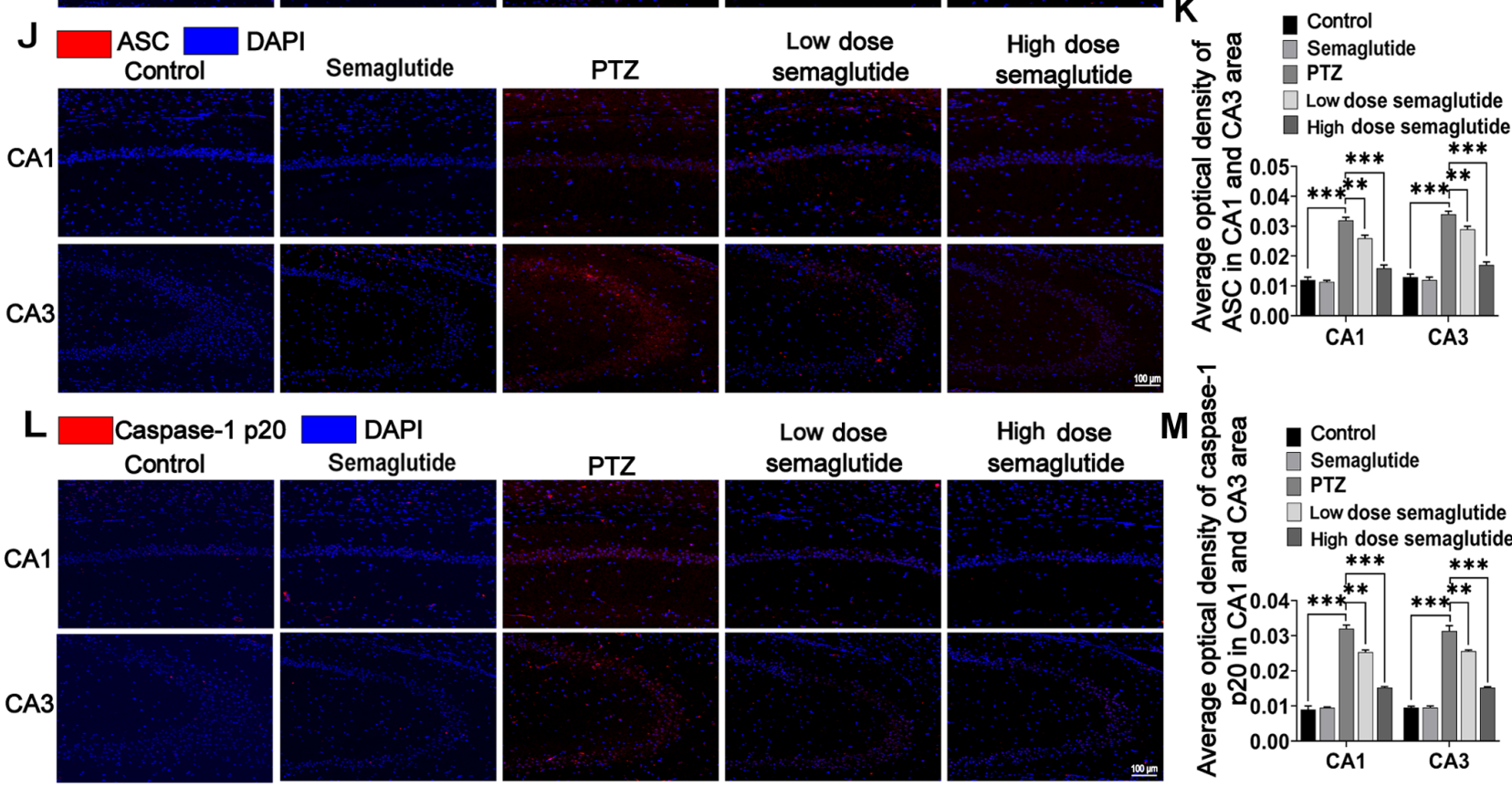

d

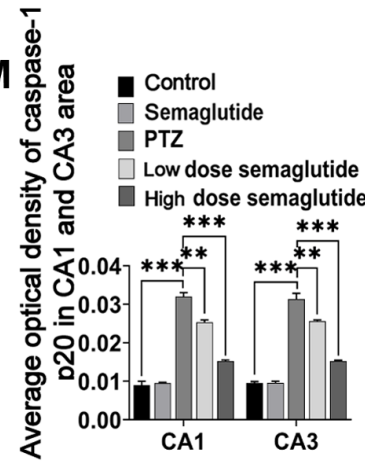

Figure 7. Semaglutide affects NLRP3 inflammasome activation. Reverse transcription-quantitative PCR analysis results showing that both the 10 and $25 \mathrm{nM} / \mathrm{kg}$ semaglutide reduced the mRNA levels of (A) NLRP3, (B) ASC and (C) caspase-1 p20 (one-way ANOVA). (D) Representative images and (E-G) statistical results of immunoblot analysis showing that both 10 and $25 \mathrm{nM} / \mathrm{kg}$ semaglutide reduced the band intensities of the NLRP3 inflammasome (one-way ANOVA). (H-M) Representative immunofluorescence images and densitometric analysis showing that both doses of semaglutide (10 and $25 \mathrm{nM} / \mathrm{kg}$ ) lowered the fluorescence intensities in the CA1 and CA3 regions (one-way ANOVA). Data are presented as the means \pm SD. ${ }^{*} \mathrm{P}<0.05,{ }^{* * *} \mathrm{P}<0.01$ and ${ }^{* * * *} \mathrm{P}<0.001 . \mathrm{N} \geq 3 . \mathrm{NLRP} 3, \mathrm{NLR}$ family pyrin domain containing 3; ASC, apoptosis-associated speck-like protein; PTZ, pentylenetetrazole.

high-dose semaglutide mice $(\mathrm{P}<0.001)$ to the control and semaglutide mice $(\mathrm{P}>0.05)$. The immunofluorescence intensities of
ASC and caspase- 1 p20 successively decreased from the PTZ mice (Fig. 7J-M; $\mathrm{P}<0.001$ ) to the low-dose semaglutide mice 
A

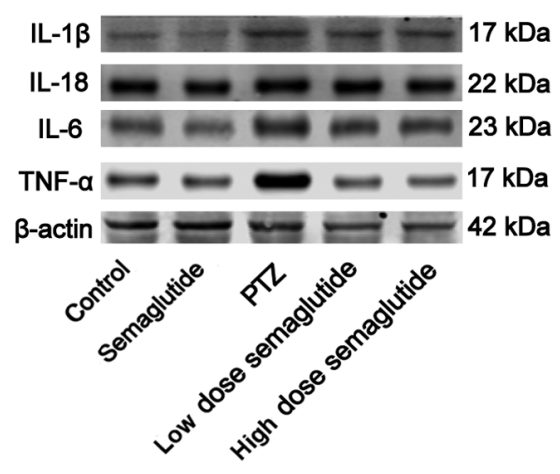

B

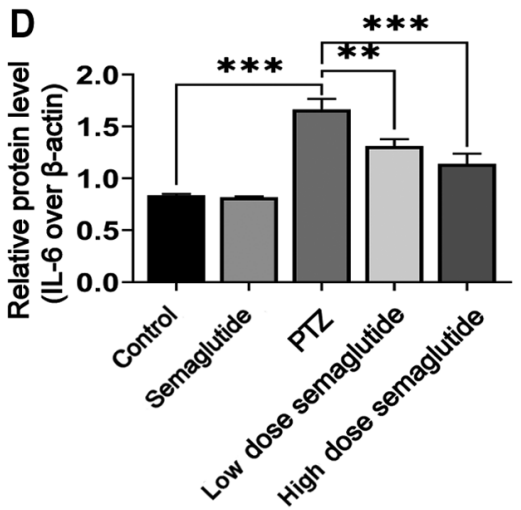

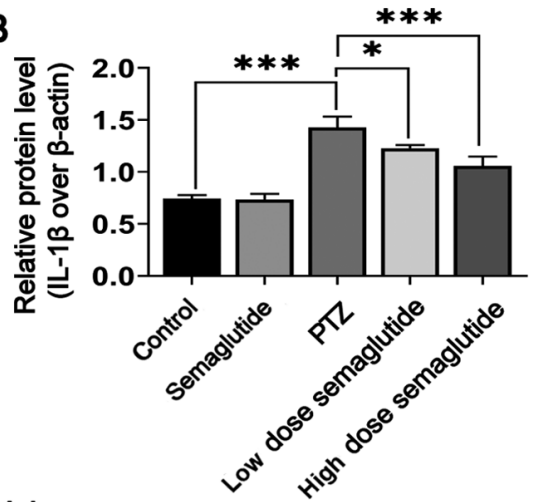

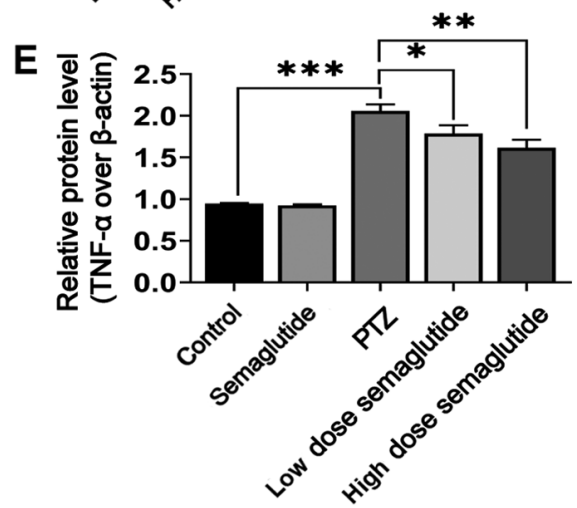

Figure 8. (A) Representative images and (B-E) statistical results of immunoblot analysis showing that both 10 and $25 \mathrm{nM} / \mathrm{kg}$ semaglutide reduced the band intensities of IL-1 $\beta$, IL-18, IL-6 and TNF- $\alpha$ in hippocampal tissues (one-way ANOVA). Data are presented as the means $\pm \mathrm{SD}$. ${ }^{*} \mathrm{P}<0.05,{ }^{* *} \mathrm{P}<0.01,{ }^{* * *} \mathrm{P}<0.001$. $\mathrm{N} \geq 3$. PTZ, pentylenetetrazole.

$(\mathrm{P}<0.01)$ to the high-dose semaglutide mice $(\mathrm{P}<0.001)$ to the control and semaglutide mice $(\mathrm{P}>0.05)$. These results showed that semaglutide alleviated hippocampal neuronal insult and cognitive impairment in mice, possibly via suppression of NLRP3 inflammasome activation.

Semaglutide decreases inflammatory cytokine secretion in PTZ-kindled mice. In various respects, inflammasome activation contributes to the release of inflammatory cytokines, which play a pivotal role in epileptogenesis $(14,15,38)$. The present study demonstrated that semaglutide blocked NLRP3 inflammasome activation. Subsequently, WB was used to detect the protein levels of inflammatory cytokines in the hippocampus of PTZ-kindled mice. Inflammatory cytokine release were increased in the PTZ mice with respect to the controls (Fig. 8A-E; P<0.001). Nonetheless, the expression levels of the inflammatory cytokines IL-1 $\beta$, IL-18, IL-6 and TNF- $\alpha$ were partially downregulated in the low-dose (Fig. 8B, $\mathrm{P}<0.05$; Fig. $8 \mathrm{C}, \mathrm{P}<0.05$; Fig. $8 \mathrm{D}, \mathrm{P}<0.01$; Fig. $8 \mathrm{E}, \mathrm{P}<0.05$, respectively) and high-dose semaglutide mice with respect to the $\mathrm{PTZ}$ mice $(\mathrm{P}<0.001, \mathrm{P}<0.01, \mathrm{P}<0.001$ and $\mathrm{P}<0.01$, respectively), though expression levels between the semaglutide alone mice and the controls were not significantly different. These results indicated that semaglutide also blocked the secretion of inflammatory cytokines in PTZ-kindled mice.

\section{Discussion}

In the present study, semaglutide was found to alleviate the LPS-and nigericin-mediated inflammatory response and
LDH release by inhibiting NLRP3 inflammasome activation in vitro. Semaglutide was also shown to decrease kindling rate and seizure severity in vivo, and to alleviate hippocampal neuronal injury and cognitive dysfunction in PTZ-kindled mice. Furthermore, the results showed that semaglutide blocked NLRP3 inflammasome activation and decreased inflammatory cytokine release in the hippocampal tissues of PTZ-kindled mice. These findings indicated that semaglutide reduced seizure severity, exerted neuroprotective effects and ameliorated cognitive dysfunction, possibly by inhibiting NLRP3 inflammasome activation and decreasing inflammatory cytokine secretion (Fig. 9).

These findings were in line with previous studies indicating that GLP-1 analogues reduce tissue apoptosis or pyroptosis by inhibiting NLRP3 activation $(21,39)$. Based on these in vitro findings, a chronic epilepsy model was established using PTZ-kindled mice. In vivo, preventive administration of either 10 or $25 \mathrm{nM} / \mathrm{kg}$ semaglutide every other day decreased seizure severity in PTZ-kindled mice. This result was similar to the results of daily injection of liraglutide and sitagliptin in previous studies $(25,40)$.

Epilepsy is frequently accompanied by cognitive dysfunction (41). Indeed; learning impairment and cognitive dysfunction have been observed in patients with epilepsy and various animal models, and even if the seizures were controlled, the cognitive dysfunction was not markedly improved $(11,41)$. Therefore, after finding that semaglutide decreased seizure severity in PTZ-kindled mice, behavioral tests were used in the current study to assess whether semaglutide ameliorated cognitive dysfunction as a result of PTZ kindling. In the NOR, 


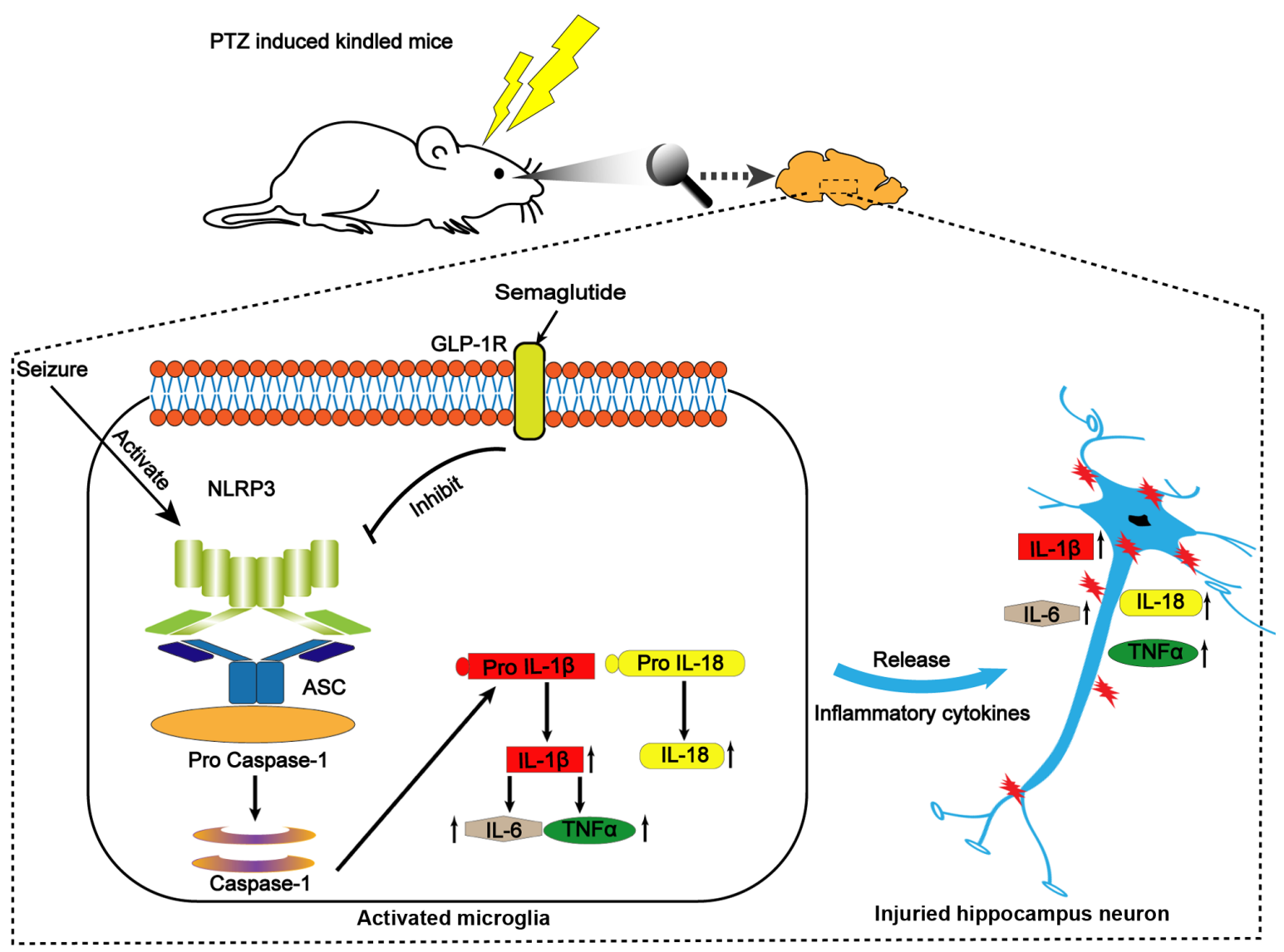

Figure 9. Hypothetical mechanism of semaglutide action in PTZ-kindled mice. PTZ, pentylenetetrazole; NLRP3, NLR family pyrin domain containing 3; ASC, apoptosis-associated speck-like protein; GLP-1R, glucagon like peptide-1.

shuttle box and MWM tests, both 10 and $25 \mathrm{nM} / \mathrm{kg}$ semaglutide ameliorated cognitive dysfunction in PTZ-kindled mice. This result was consistent with previous findings in cerebral ischemia (27), AD (28) and PD (29) models. The results showed that semaglutide ameliorated cognitive dysfunction in CNS diseases, including epilepsy. However, in the NOR test, the DI of the low-dose semaglutide mice showed an increasing trend with respect to that in the PTZ mice $(\mathrm{P}>0.05)$, but the DI in the high-dose semaglutide mice was significantly higher with respect to that in the PTZ mice $(\mathrm{P}<0.01)$. These results may be caused by the difference in the semaglutide dose; the $25 \mathrm{nM} / \mathrm{kg}$ dose may more effectively ameliorate cognitive dysfunction than the $10 \mathrm{nM} / \mathrm{kg}$ dose in PTZ-kindled mice.

Numerous lines of evidence indicate that the hippocampal $\mathrm{CA} 1$ and CA 3 areas are more vulnerable to neuronal injury in patients with epilepsy. Neuronal injury further promoted learning and memory impairment (20), and hippocampal CA3 neurons are more sensitive to epilepsy-related excitotoxicity than CA1 neurons (42). Seizures aggravate injury to hippocampal neurons and cause cognitive dysfunction. Multiple GLP-1 analogues have been shown to exert neuroprotective and nerve regenerative functions under pathological conditions in previous epilepsy studies $(25,36,40)$. Our previous research showed that semaglutide reduced seizure severity and ameliorated cognitive dysfunction; thus, in the present study, whether semaglutide improved hippocampal neuronal apoptosis was further investigated. The results suggested that both semaglutide doses ameliorated neuronal injury in the hippocampal tissue, decreased the level of the apoptosis-related protein active caspase- 3 and increased the Bcl-2/Bax ratio in hippocampal neurons in PTZ-kindled mice. These results suggested that the ameliorative effect of semaglutide on cognitive dysfunction may be associated with reduced hippocampal neuronal apoptosis and enhanced nerve function recovery in PTZ-kindled mice. These effects were similar to those of semaglutide in other central nervous system diseases $(28,29)$.

Accumulating evidence has demonstrated that neuroinflammation plays a pivotal role in the pathological mechanism of epilepsy $(12,13)$. Activation of the NLRP3 inflammasome induces the secretion of IL-1 $\beta$ and IL-18 (38), and IL-1 $\beta$ further induces the release of IL-6 and TNF- $\alpha$ (43). Both inflammasomes and neuroinflammation affect the occurrence and development of epilepsy, and anti-inflammasome and anti-neuroinflammation therapies may be novel treatments for epilepsy (16). In the present study, semaglutide attenuated NLRP3 inflammasome activation and inflammatory cytokine secretion in LPS- and nigericin-induced inflammatory BV2 cells. Therefore, semaglutide may also possess anti-inflammatory effects in vivo. In PTZ-kindled mice, both doses of semaglutide (10 and $25 \mathrm{nM} / \mathrm{kg}$ ) decreased NLRP3 inflammasome activation and lowered inflammatory cytokine secretion in the present study. These results indicated that semaglutide reduced neuronal apoptosis, ameliorated cognitive dysfunction and attenuated seizure severity, possibly via an inhibitory effect on the NLRP3 inflammasome and inflammatory cytokines. 
However, the precise mechanisms by which semaglutide affects NLRP3 inflammasome signaling are unclear. Previous studies have shown that GLP-1 agonists inhibit NLRP3 inflammasome activation through sirtuin 1 signaling to alleviate cardiovascular impairment $(44,45)$. Another study found that GLP-1 agonists alleviated inflammation and attenuated peripheral nerve injury via the $\mathrm{p} 38 \mathrm{MAPK} / \mathrm{NF}-\kappa \mathrm{B}$ pathway (46). A previous study also indicated that ibuprofen has antiepileptic and neuroprotective effects through the cyclooxygenase-2/NLRP3 pathway in PTZ-kindled mice (18). However, further research is necessary to clarify the precise mechanisms.

In conclusion, through in vitro experimentation, the present study revealed that semaglutide blocked NLRP3 inflammasome activation, inhibited inflammatory cytokine secretion, and reduced LDH release. In an in vivo experiment, semaglutide was found to decrease seizure severity, reduce neuronal damage and ameliorate cognitive impairment, potentially via inhibition of NLRP3 inflammasome activation and related cascade reactions. These results offer novel insights into the treatment of epilepsy and indicate that semaglutide may be a promising adjuvant therapeutic.

\section{Acknowledgements}

The authors are grateful to the Ningxia Key Laboratory of Cerebrocranial Disease of Ningxia Medical University for their kind help on technical expertise.

\section{Funding}

The present study was supported by the National Natural Science Foundation of China (grant no. 81971085), the Ningxia Hui Autonomous Region '13th Five-Year Plan' Major Science and Technology Projects (grant no. 2016BZ07), and the Advantages Discipline Group Project of Ningxia Medical University grant (grant no. XY201511).

\section{Availability of data and materials}

The datasets used and/or analyzed during the current study are available from the corresponding author on reasonable request.

\section{Authors' contributions}

TS and FW designed the study. LW, JD and CZ conducted the experiments. LW, JD and CZ wrote the paper. BG, WY, WH, XL, YW and WL analyzed the data. LW and TS confirm the authenticity of all the raw data. All authors have read and approved the final manuscript.

\section{Ethics approval and consent to participate}

The animal study was reviewed and approved by institutional Animal Care and Use Committee of Ningxia Medical University [IACUC Animal Use Certificate No. SCXK (Ning) 2019-203].

\section{Patient consent for publication}

Not applicable.

\section{Competing interests}

The authors declare that they have no competing interests.

\section{References}

1. Rong S, Wan D, Fan Y, Liu S, Sun K, Huo J, Zhang P, Li X, Xie X, Wang F, et al: Amentoflavone affects epileptogenesis and exerts neuroprotective effects by inhibiting NLRP3 inflammasome. Front Pharmacol 10: 856, 2019.

2. Juvale IIA and Che Has AT: Possible interplay between the theories of pharmacoresistant epilepsy. Eur J Neurosci 53: 1998-2026, 2021.

3. Xiao D, Lv J, Zheng Z, Liu Y, Zhang Y, Luo C, Qi L, Qin B and Liu C: Mechanisms of microRNA 142 in mitochondrial autophagy and hippocampal damage in a rat model of epilepsy. Int J Mol Med 47: 98, 2021.

4. González-H G, Contreras-García IJ, Sánchez-Huerta K, Queiroz CM, Gallardo Gudiño LR, Mendoza-Torreblanca JG and Zamudio SR: Levetiracetam reduced the basal excitability of the dentate gyrus without restoring impaired synaptic plasticity in rats with temporal lobe epilepsy. Brain Sci 10: E634, 2020.

5. Guiard BP and Di Giovanni G: Central serotonin-2A (5-HT2A) receptor dysfunction in depression and epilepsy: The missing link? Front Pharmacol 6: 46, 2015.

6. Fisher RS, van Emde Boas W, Blume W, Elger C, Genton P, Lee P and Engel J Jr: Epileptic seizures and epilepsy: Definitions proposed by the International League Against Epilepsy (ILAE) and the International Bureau for Epilepsy (IBE). Epilepsia 46: 470-472, 2005.

7. Baroli G, Sanchez JR, Agostinelli E, Mariottini P and Cervelli M: Polyamines: The possible missing link between mental disorders and epilepsy (Review). Int J Mol Med 45: 3-9, 2020.

8. Perucca E, Brodie MJ, Kwan P and Tomson T: 30 years of second-generation antiseizure medications: Impact and future perspectives. Lancet Neurol 19: 544-556, 2020.

9. Wang Y and Chen Z: An update for epilepsy research and antiepileptic drug development: Toward precise circuit therapy. Pharmacol Ther 201: 77-93, 2019.

10. Löscher W, Potschka H, Sisodiya SM and Vezzani A: Drug resistance in epilepsy: Clinical impact, potential mechanisms, and new innovative treatment options. Pharmacol Rev 72: 606-638, 2020.

11. West PJ, Saunders GW, Remigio GJ, Wilcox KS and White HS: Antiseizure drugs differentially modulate $\theta$-burst induced long-term potentiation in C57BL/6 mice. Epilepsia 55: 214-223, 2014.

12. Vezzani A, Fujinami RS, White HS, Preux PM, Blümcke I, Sander JW and Löscher W: Infections, inflammation and epilepsy. Acta Neuropathol 131: 211-234, 2016.

13. Espinosa-Garcia C, Zeleke $\mathrm{H}$ and Rojas A: Impact of stress on epilepsy: Focus on neuroinflammation-A mini review. Int $\mathrm{J}$ Mol Sci 22: 4061, 2021.

14. Shao BZ, Xu ZQ, Han BZ, Su DF and Liu C: NLRP3 inflammasome and its inhibitors: A review. Front Pharmacol 6: 262, 2015.

15. Wu C, Zhang G, Chen L, Kim S, Yu J, Hu G, Chen J, Huang Y, Zheng $\mathrm{G}$ and Huang S: The role of NLRP3 and IL-1 $\beta$ in refractory epilepsy brain injury. Front Neurol 10: 1418, 2020.

16. de Brito Toscano EC, Vieira ÉL, Dias BB, Caliari MV, Gonçalves AP, Giannetti AV, Siqueira JM, Suemoto CK, Leite RE, Nitrini R, et al: NLRP3 and NLRP1 inflammasomes are up-regulated in patients with mesial temporal lobe epilepsy and may contribute to overexpression of caspase-1 and IL- $\beta$ in sclerotic hippocampi. Brain Res 1752: 147230, 2021.

17. Kegler A, Caprara AL, Pascotini ET, Arend J, Gabbi P, Duarte MM, Furian AF, Oliveira MS, Royes LF and Fighera MR: Apoptotic markers are increased in epilepsy patients: A relation with manganese superoxide dismutase Ala16Val polymorphism and deizure type through IL-1 $\beta$ and IL- 6 pathways. BioMed Res Int 2020: 6250429, 2020.

18. Liu R, Wu S, Guo C, Hu Z, Peng J, Guo K, Zhang X and Li J: Ibuprofen exerts antiepileptic and neuroprotective effects in the rat model of pentylenetetrazol-induced epilepsy via the COX-2/ NLRP3/IL-18 pathway. Neurochem Res 45: 2516-2526, 2020.

19. Tran KL, Park YI, Pandya S, Muliyil NJ, Jensen BD, Huynh K and Nguyen QT: Overview of glucagon-like peptide-1 receptor agonists for the treatment of patients with type 2 diabetes. Am Health Drug Benefits 10: 178-188, 2017. 
20. Koshal P, Jamwal S, Kumar P. Glucagon-like Peptide-1 (GLP-1) and neurotransmitters signaling in epilepsy: An insight review. Neuropharmacology 136: 271-279, 2018.

21. Zhu W, Feng PP, He K, Li SW and Gong JP: Liraglutide protects non-alcoholic fatty liver disease via inhibiting NLRP3 inflammasome activation in a mouse model induced by high-fat diet. Biochem Biophys Res Commun 505: 523-529, 2018.

22. Yu X, Hao M, Liu Y, Ma X, Lin W, Xu Q, Zhou H, Shao N and Kuang H: Liraglutide ameliorates non-alcoholic steatohepatitis by inhibiting NLRP3 inflammasome and pyroptosis activation via mitophagy. Eur J Pharmacol 864: 172715, 2019.

23. Cork SC, Richards JE, Holt MK, Gribble FM, Reimann F and Trapp S: Distribution and characterisation of Glucagon-like peptide-1 receptor expressing cells in the mouse brain. Mol Metab 4: 718-731, 2015

24. Graham DL, Durai HH, Trammell TS, Noble BL, Mortlock DP, Galli A and Stanwood GD: A novel mouse model of glucagon-like peptide-1 receptor expression: A look at the brain. J Comp Neurol 528: 2445-2470, 2020.

25. Liu S, Jin Z, Zhang Y, Rong S, He W, Sun K, Wan D, Huo J, Xiao L, Li X, et al: The glucagon-like peptide-1 analogue liraglutide reduces seizures susceptibility, cognition dysfunction and neuronal apoptosis in a mouse model of dravet syndrome. Front Pharmacol 11: 136, 2020.

26. Dhillon S: Semaglutide: First global approval. Drugs 78: 275-284, 2018.

27. Yang X, Feng $P$, Zhang X, Li D, Wang R, Ji C, Li G and Hölscher C: The diabetes drug semaglutide reduces infarct size, inflammation, and apoptosis, and normalizes neurogenesis in a rat model of stroke. Neuropharmacology 158: 107748, 2019.

28. Chang YF, Zhang D, Hu WM, Liu DX and Li L: Semaglutidemediated protection against $A \beta$ correlated with enhancement of autophagy and inhibition of apotosis. J Clin Neurosci 81 234-239, 2020

29. Zhang L, Zhang L, Li L and Hölscher C: Semaglutide is neuroprotective and reduces $\alpha$-synuclein levels in the chronic MPTP mouse model of Parkinson's disease. J Parkinsons Dis 9: 157-171, 2019.

30. Minkeviciene R, Rheims S, Dobszay MB, Zilberter M, Hartikainen J, Fülöp L, Penke B, Zilberter Y, Harkany T, Pitkänen A, et al: Amyloid beta-induced neuronal hyperexcitability triggers progressive epilepsy. J Neurosci 29: 3453-3462, 2009.

31. Yan XX, Cai Y, Shelton J, Deng SH, Luo XG, Oddo S, Laferla FM, Cai H, Rose GM and Patrylo PR: Chronic temporal lobe epilepsy is associated with enhanced Alzheimer-like neuropathology in 3xTg-AD mice. PLoS One 7: e48782, 2012.

32. Nam HY, Nam JH, Yoon G, Lee JY, Nam Y, Kang HJ, Cho HJ, Kim J and Hoe HS: Ibrutinib suppresses LPS-induced neuroinflammatory responses in BV2 microglial cells and wild-type mice. J Neuroinflammation 15: 271, 2018.

33. Zeng QZ, Yang F, Li CG, Xu LH, He XH, Mai FY, Zeng CY, Zhang CC, Zha QB and Ouyang DY: Paclitaxel Enhances the Innate Immunity by Promoting NLRP3 Inflammasome Activation in Macrophages. Front Immunol 10: 72, 2019.
34. Koshal P and Kumar P: Neurochemical modulation involved in the beneficial effect of liraglutide, GLP-1 agonist on PTZ kindling epilepsy-induced comorbidities in mice. Mol Cell Biochem 415: 77-87, 2016.

35. Livak KJ and Schmittgen TD: Analysis of relative gene expression data using real-time quantitative PCR and the 2(-Delta Delta $\mathrm{C}(\mathrm{T})$ ) method. Methods 25: 402-408, 2001.

36. Zheng Z, Liang P, Hou B, Lu X, Ma Q, Yu X, Han S, Peng B, Chen T, Liu W, et al: The effect of dipeptidyl peptidase IV on disease-associated microglia phenotypic transformation in epilepsy. J Neuroinflammation 18: 112, 2021.

37. Vezzani A, Balosso S and Ravizza T: Neuroinflammatory pathways as treatment targets and biomarkers in epilepsy. Nat Rev Neurol 15: 459-472, 2019.

38. Swanson KV, Deng M and Ting JP: The NLRP3 inflammasome: Molecular activation and regulation to therapeutics. Nat Rev Immunol 19: 477-489, 2019.

39. Birnbaum Y, Tran D, Bajaj M and Ye Y: DPP-4 inhibition by linagliptin prevents cardiac dysfunction and inflammation by targeting the Nlrp3/ASC inflammasome. Basic Res Cardiol 114: 35,2019 .

40. Safar MM, Shahin NN, Mohamed AF and Abdelkader NF: Suppression of BACE1 and amyloidogenic/RAGE axis by sitagliptin ameliorates PTZ kindling-induced cognitive deficits in rats. Chem Biol Interact 328: 109144, 2020.

41. Zhu L, Chen L, Xu P, Lu D, Dai S, Zhong L, Han Y, Zhang M, Xiao B, Chang L, et al: Genetic and molecular basis of epilepsy-related cognitive dysfunction. Epilepsy Behav 104: $106848,2020$.

42. Lai MC, Lin KM, Yeh PS, Wu SN and Huang CW: The novel effect of immunomodulator-glatiramer acetate on epileptogenesis and epileptic seizures. Cell Physiol Biochem 50: 150-168, 2018.

43. Alomar SY, Gentili A, Zaibi MS, Kepczyńska MA and Trayhurn P: IL-1 $\beta$ (interleukin-1 $\beta$ ) stimulates the production and release of multiple cytokines and chemokines by human preadipocytes. Arch Physiol Biochem 122: 117-122, 2016.

44. Chen A, Chen Z, Xia Y, Lu D, Yang X, Sun A, Zou Y, Qian J and Ge J: Liraglutide attenuates NLRP3 inflammasome-dependent pyroptosis via regulating SIRT1/NOX4/ROS pathway in H9c2 cells. Biochem Biophys Res Commun 499: 267-272, 2018.

45. Luo X, Hu Y, He S, Ye Q, Lv Z, Liu J and Chen X: Dulaglutide inhibits high glucose-induced endothelial dysfunction and NLRP3 inflammasome activation. Arch Biochem Biophys 671: 203-209, 2019.

46. Ma J, Shi M, Zhang X, Liu X, Chen J, Zhang R, Wang X and Zhang $\mathrm{H}$ : GLP 1R agonists ameliorate peripheral nerve dysfunction and inflammation via $\mathrm{p} 38 \mathrm{MAPK} / \mathrm{NF}-\kappa \mathrm{B}$ signaling pathways in streptozotocin induced diabetic rats. Int $\mathrm{J}$ Mol Med 41: 2977-2985, 2018.

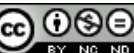

This work is licensed under a Creative Commons Attribution-NonCommercial-NoDerivatives 4.0 International (CC BY-NC-ND 4.0) License. 